\title{
Neutral beam stopping and emission in fusion plasmas I: deuterium beams
}

\author{
H Anderson $\dagger$, M G von Hellermann $\ddagger$, R Hoekstra§, L D Horton $\ddagger$, \\ A C Howman $\ddagger$, R W T Konig $\ddagger$, R Martin $\dagger$, R E Olson $\|$ and H P Summers $\dagger$ \\ $\dagger$ Department of Physics and Applied Physics, University of Strathclyde, Glasgow G4 0NG, UK \\ \$ JET Joint Undertaking, Abingdon, Oxon OX14 3EA, UK \\ $\S$ KVI Atomic Physics, Zernikelaan 25, 9747 AA Groningen, Netherlands \\ || Physics Department, University of Missouri, Rolla MO 65401, USA
}

Received 10 January 2000, in final form 4 May 2000

\begin{abstract}
The charge transfer reaction of neutral deuterium beams with impurities enables one of the principle quantitative diagnostic measurements of the hot core fusion plasma; that is, charge exchange spectroscopy. The complementary measurement of beam emission spectroscopy has been fruitful in motional Stark wavelength shift and fluctuation studies, but less so in using absolute measured intensities. In the last two years we have achieved substantial improvement in the quantitative analysis and agreement between the observed and modelled beam emission at the JET Joint Undertaking. This has depended on improved spectral fitting of the overlayed $\mathrm{D}_{\alpha}$ motional Stark multiplet, self-consistent beam emission and impurity charge exchange modelling and analysis, and revision of the data entering the modelling of the beam emission process. The paper outlines the present JET beam emission diagnostic system and the collisional radiative modelling of deuterium beam stopping and emission. The nature and organization of the effective derived data directly used in experimental interpretation at JET are described and some results of spectral analysis of deuterium beam emission given. The practical implementation of the methods described here is part of the ADAS Project.
\end{abstract}

\section{Introduction}

In addition to heating and fuelling tokamak plasmas, neutral deuterium beam injection provides an excellent diagnostic to investigate the concentration of impurities in the confined plasma via charge exchange spectroscopy (Isler 1994). For its use a detailed knowledge of the beam attenuation or alternatively the neutral beam density is required. There are two methods which may be employed to determine the neutral beam density at points along the beam path. The first, and in principle the more accurate method, involves the direct measurement of the intensity of the $\mathrm{D}_{\alpha}$ light emitted from the excited beam atoms as they traverse the plasma. Then with the use of atomic modelling to evaluate effective $\mathrm{D}_{\alpha}$ emission coefficients, the local neutral beam density can be recovered. This is the basis of beam emission spectroscopy (Boileau et al 1989a). The second method, a numerical calculation of the attenuation along the beam path from the point of injection, has received most attention due to the ease with which it can be implemented for inter-pulse experimental analysis. This calculation takes into consideration the atomic processes that contribute to ionizing the beam neutrals and has been the subject of many investigations (Riviere 1971, Boley et al 1984, Boileau et al 1989b, Korotkov and Samsonov 1989, Janev et al 1989, Suzuki et al 1998). In these studies the attenuation of the 
beam is characterized by effective beam stopping coefficients (or cross sections) that represent the rate at which the beam atoms are ionized.

In high-density plasmas, such as in the anticipated operating regime of the next generation of tokamaks, the accurate deduction of the neutral beam density can only be achieved with confidence using the spectroscopic method. This is because the enhanced attenuation amplifies uncertainties in the fundamental atomic data and so limits the accuracy with which the neutral beam density can be determined by calculation (von Hellermann et al 1995, Mandl et al 1993). This fact alone motivated a long-term study aimed at exploiting the $\mathrm{D}_{\alpha}$ beam emission feature at the JET Joint Undertaking and improving the accuracy of the spectroscopic method.

In practice, this has proved difficult to achieve for several reasons. First, the observed $\mathrm{D}_{\alpha}$ emission is distorted due to the motional Stark effect and substantial Doppler shifts in the oblique viewing lines (see section 2). Also, the observed $\mathrm{D}_{\alpha}$ Stark multiplet is in fact a superposition of such features from different injectors and from different energy fractions within each individual injector. At the JET Joint Undertaking, each injector assembly comprises two vertical banks, a normal and a tangential bank. Each bank contains four individual injectors and in each injector there are half and third fractional energy components (35 keV/amu and $23 \mathrm{keV} / \mathrm{amu}$ ) as well as the JET primary $70 \mathrm{keV} / \mathrm{amu}$ component. The resulting composite $\mathrm{D}_{\alpha}$ spectral emission contains more than 150 components and dissection of the spectral region has required a substantial improvement in the technique of spectral fitting. Second, much of the fundamental ion-atom collisional cross section data required to adequately model the excited beam atom populations has until recently been either unavailable or of low accuracy. Third, the deduction of the local beam density (from calculated stopping or measured beam emission) and of the local impurity densities (from charge exchange spectroscopy) must be performed self-consistently and in a time scale suitable for inter-pulse analysis. This has involved automating the matching of various observations and their merging with additional JET diagnostic signals, including radial profiles of electron temperature and density.

In this paper we report recent advances in the spectroscopic deduction of the line integrated neutral beam density at JET. The evaluation of the neutral beam density using the attenuation calculation is also considered. To achieve the former and latter we employ a bundled- $n$ collisional-radiative model to evaluate the effective beam stopping and $\mathrm{D}_{\alpha}$ beam emission coefficients. The calculations of accurate effective beam stopping and $\mathrm{D}_{\alpha}$ emission coefficients are in fact closely linked. This is because at the densities of the fusion plasma core, the collision limit for neutral deuterium is around $n=4$. Thus the population of the $n=3$ quantum shell of neutral deuterium is influenced by collisional excitation and ionization losses and this in turn influences the effective stopping coefficient. The collisional-radiative model is summarized in section 4 with details of the key fundamental collision data inputs in section 5 . The computational implementation is described in section 6 and the sensitivity of the derived coefficients to error in the fundamental data is examined in section 7 . The experimental aspects associated with the beam emission spectroscopy diagnostic at the JET Joint Undertaking are summarized in section 3 and a comparison between the measured and calculated line integrated beam densities is given in section 8 . The modelling codes and data sets used in this work form part of the Atomic Data and Analysis Structure, ADAS (Summers 1994, 1999).

\section{The observed $\mathbf{D}_{\alpha}$ beam emission feature}

The primary beam emission observation is of a series of overlapped Doppler shifted Stark multiplet features (see for example von Hellermann et al 1995). The existence of three fractional energy components in each diagnostic injector gives rise to three Stark multiplet 


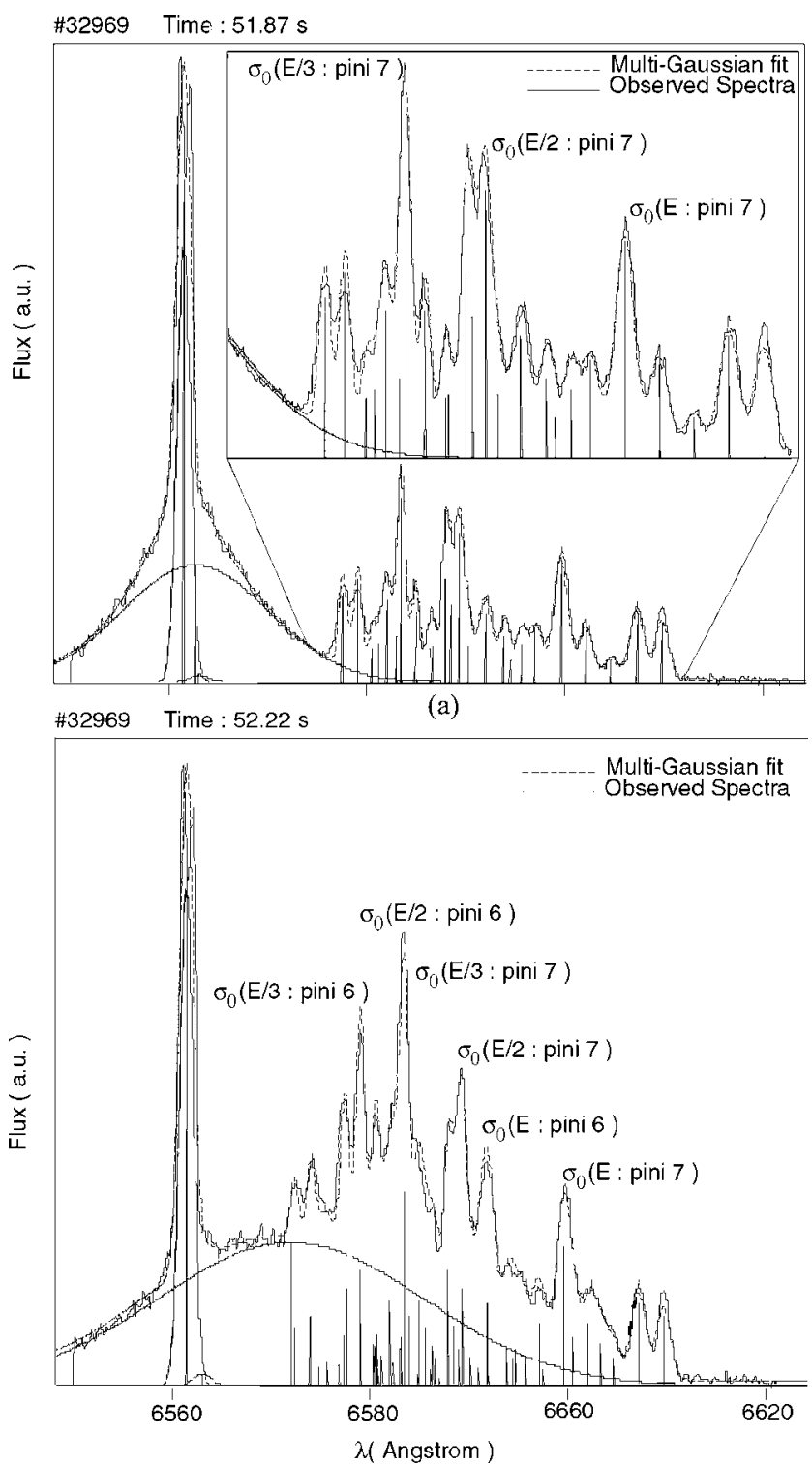

(b)

Figure 1. Complexity of the beam emission signature: (a) shows the beam emission feature during a single bank period of the pulse 32969 , and (b) illustrates the increasing complexity of the observed spectrum during the double beam bank period.

features. Each Stark multiplet is Doppler shifted according to the velocity of the relevant energy component and the viewing angle between the neutral injector and the line of sight. The salient features of the spectrum as we move in the direction of increasing wavelength (see figure 1(a)) commences with the large $\mathrm{D}_{\alpha}$ edge emission line situated at $6561 \AA$, originating from thermal neutral deuterium between the last-closed magnetic flux surface and the vessel wall. Next we encounter a cluster of Stark multiplet features which are collectively known as the beam emission spectrum. Each Stark multiplet feature consists of nine observable Stark 
components (up to $\pm \pi 4$ ). The higher-order components of the Stark resolved spectrum are too weak in intensity to observe reliably. The relative positions and intensities of the features of the beam emission spectrum are highly constrained theoretically. The local magnetic field orthogonal to the neutral beam particle path together with precisely known velocities of the energy fractions determines the wavelength separation of the Stark components. The relative intensities of the $\sigma$ and $\pi$ polarization components are also determined since Stark fine structure populations are fully mixed at the JET core plasma densities $\left(>2 \times 10^{19} \mathrm{~m}^{-3}\right)$ and the geometry is specified. In the case of double beam bank pulses, which are the more common pulse type at JET, the recorded beam emission spectrum originates from the excited beam neutrals of two injectors (6 and 7 of octant 8 ) so that the spectrum comprises of six overlapping Stark multiplet features. In figure 1(a), the beam emission spectrum during the single bank period of the pulse No 32969 shows the three Stark multiplets, but this increases in complexity during the double beam bank period of the pulse as shown in figure 1(b). In both the single and double beam bank spectra, additional spectral lines in the vicinity of the beam emission spectrum can also be observed. A detailed description of the full single bank spectrum is given by Mandl et al (1993).

\section{Experimental spectral data handling}

The beam emission spectrum is recorded using a back illuminated CCD camera. A fan of 12 fibre optics are focused along the trajectory associated with injectors 6 and 7 of octant 8 from the diagnostic port illustrated in figure 2. Injectors 6 and 7 are members of the normal and tangential banks, respectively, and share a similar trajectory. Each of the 12 fibre optics are employed to relay the $\mathrm{D}_{\alpha}$ emission feature back to a Czerny-Turner-type spectrometer, equipped with a two-dimensional CCD camera. The signals are recorded by the camera every $50 \mathrm{~ms}$ and stored awaiting analysis. Simultaneous charge exchange emission spectroscopic measurements are made. These have been described on a number of occasions (e.g. Mandl et al 1993). Two independent spectrometers similar to the beam emission spectrometer above monitor the $\mathrm{C}^{5+}(5291 \AA), \mathrm{He}^{+}(4685 \AA)$ and the $\mathrm{Be}^{3+}(4658 \AA)$ charge exchange spectrum. In addition, a single central vertical line of sight feeds light to two spectrometers, monitoring the $\mathrm{C}^{5+}, \mathrm{He}^{+}$and $\mathrm{Be}^{3+}$ spectra. This ensures that the dominant light impurities are measured so they can be used as input in the neutral beam density analysis.

The reduction of the beam emission spectrum then occurs in two stages. The first stage involves the use of a generalized multi-Gaussian spectral analysis code to extract the total flux associated with each Stark multiplet. The second stage is a post-processing step. The post-processing code, which is known as the Charge Exchange Analysis Package (CHEAP), evaluates both the line integrated neutral beam density and the impurity concentrations using the beam emission flux measurements and charge exchange spectroscopy measurements.

For the present work it was necessary to extend the capabilities of the spectral analysis code to accommodate the increase in complexity of the observed spectra associated with double beam bank pulses. The original code, as described by Mandl et al (1993), was written primarily for the analysis of single beam bank spectra. We also took this opportunity to improve the accuracy of the fitting procedure by introducing nonlinear constraints, in addition to updating the algorithm employed to optimize the array of Gaussians used to produce the best-fit synthetic spectrum.

The improved spectral analysis code operates by first locating the position of the $\mathrm{D}_{\alpha}$ edge emission line. Since this emission originates from the edge of the vessel, it is free from any Doppler shift due to bulk motion of the plasma and is used as a wavelength reference. Then the position of the Doppler shifted primary $\sigma_{0}$ Stark component associated with each Stark 

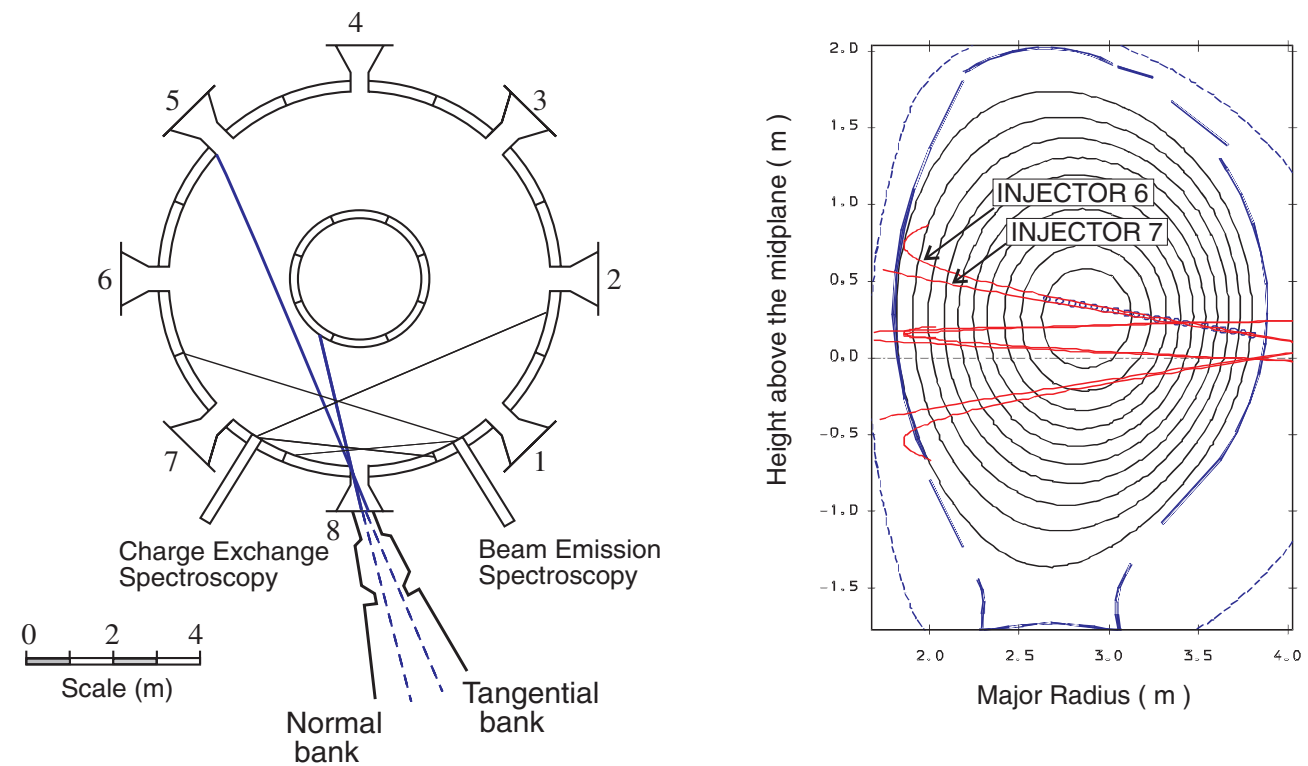

Figure 2. Plan view and side elevation of the JET tokamak. The figure on the left illustrates the location of the observation ports for charge exchange and beam emission spectroscopies. The figure on the right shows the typical trajectories of each neutral injector; particular attention should be drawn to diagnostic injectors 6 and 7, which are annotated.

multiplet is located. The position of each primary Stark component is determined by the velocity, $v_{b}$, of the relevant energy fraction and the viewing angle, $\alpha$, between the neutral injectors and the line of sight:

$$
\Delta \lambda_{\text {Doppler }}=\lambda_{0} \frac{v_{b}}{c} \cos (\alpha)
$$

where $\lambda_{0}$ is the natural wavelength. The locations of the remaining Stark components for each multiplet are identified by assuming that the Stark splitting is constant over the spectral region, as given by the expression

$$
\Delta \lambda_{\text {Stark }}=\frac{3}{2} \frac{e a_{0}}{h c} \lambda^{2}\left|E_{\mathrm{L}}\right|
$$

where $\lambda$ is the unperturbed wavelength. Estimates for the magnitude of the Lorentz electric field, $\left|E_{\mathrm{L}}\right|$, are obtained from the results of magnetic equilibrium calculations (Zwingmann et al 1997) together with knowledge of the beam velocity and experimental geometry. The positions of the components are then specified relative to the appropriate Doppler shifted primary Stark feature.

The spectral features are represented by Gaussians. The amplitudes of the Gaussians for the primary $\sigma_{0}$ Stark components are treated as free parameters, whilst the amplitudes of the Gaussians for the remaining Stark features are specified by a parametric relationship relative to the amplitude of the $\sigma_{0}$ components. This relationship is based on the intensity ratios of the individual Stark components which are entered by the user as input. The resulting parameterized array of Gaussians is then optimized to obtain a synthetic spectrum that yields the best fit to the experimentally observed data. The synthetic spectrum is then used to extract the flux from each Stark multiplet.

Since the deduction of the line integrated neutral beam density, using either the numerical attenuation calculation or the beam emission spectrum, requires knowledge of the impurity 
content of the plasma and the deduction of impurity concentrations requires the neutral beam density, an iterative approach must be used (von Hellermann and Summers 1993). The CHEAP package implements such an iterative calculation starting from initial impurity estimates. This is achieved by acquiring theoretical beam stopping coefficients from rapid-look-up tables as discussed in section 6 . The line integrated neutral beam density is then obtained using the expression

$$
\int N_{\mathrm{b}} \mathrm{d} s=N_{0} \int \exp \left(-\frac{x^{2}}{w_{x}^{2}}-\frac{y^{2}}{w_{y}^{2}}\right) \exp \left(-\int N_{\mathrm{e}} \frac{S_{\mathrm{cr}}}{v_{\mathrm{b}}} \mathrm{d} l\right) \mathrm{d} s
$$

where $\mathrm{d} l$ is along the path taken by the neutral beam, $S_{\mathrm{cr}}$ is the effective beam stopping coefficient written in terms of the electron density $N_{\mathrm{e}} . N_{0}$ is the initial beam density on entry to the plasma and $\mathrm{d} s$ is an element of length along the line of sight across the neutral beam profile. At JET the neutral beam profile can be described by a Gaussian which has a full-width half-maximum in the $x$ and $y$ directions of $w_{x}$ and $w_{y}$, respectively, where the $x$ and $y$ axes are defined to be orthogonal both to each other and to the direction of the beam.

The effective $\mathrm{D}_{\alpha}$ emission coefficients are also obtained by CHEAP from rapid-look-up tables. Then the line integrated beam density can be evaluated in the alternative way from the $\mathrm{D}_{\alpha}$ spectral emission, as given (for a single injector) by

$$
\int N_{\mathrm{b}} \mathrm{d} s=\frac{\chi}{N_{\mathrm{e}}}\left[\frac{\Phi\left(E_{0} / 3\right)}{q_{\mathrm{D}_{\alpha}}\left(E_{0} / 3\right)}+\frac{\Phi\left(E_{0} / 2\right)}{q_{\mathrm{D}_{\alpha}}\left(E_{0} / 2\right)}+\frac{\Phi\left(E_{0}\right)}{q_{\mathrm{D}_{\alpha}}\left(E_{0}\right)}\right]
$$

where $\Phi$ is the flux associated with the Stark multiplet corresponding to the full $\left(E_{0}\right)$, half $\left(E_{0} / 2\right)$ and third $\left(E_{0} / 3\right)$ fractional energy component. The quantity $q_{\mathrm{D}_{\alpha}}$ is the effective $\mathrm{D}_{\alpha}$ beam emission coefficient and $\chi$ is the window transmission factor. The latter is used to compensate for the observation window being optically degraded by the deposition of impurities and is obtained using a cross calibration procedure (Morsi et al 1995).

In principle, either method of deducing the line integrated neutral density may be employed by CHEAP, although routine analysis up to the present time has used the attenuation calculation. Note that the reduction of the impurity charge exchange signals is also achieved by accessing equivalent fast-look-up tables of theoretical charge exchange effective emission coefficients (Boileau et al 1989b, Spence and Summers 1986) and that CHEAP also makes use of bremsstrahlung measurements.

\section{The collisional-radiative model for beam stopping and emission}

We have found it advantageous to embed the calculation of beam stopping and beam emission for hydrogen in a more general picture of neutral hydrogen as a radiating, ionizing and recombining species in the fusion plasma. This is because an initial fast neutral hydrogen beam atom can lead to a high-temperature thermal hydrogen atom in a beam halo and ultimately to a lower-temperature thermal hydrogen atom at the plasma periphery. Also, with the variation of primary beam energies that occur in fusion experiments and the inevitable fractional energy components, there are a wide range of energies of hydrogen donors present, which influence the observed charge exchange impurity spectra and contribute to the overall composite $\mathrm{D}_{\alpha}$ beam emission feature. For fast beams, ground-state donors primarily drive the visible impurity charge exchange spectra, while at thermal energies it is almost entirely excited donors that do this. From the point of view of the hydrogen atom populations, the most important type of collision changes from ion impact at beam energies to electron impact in the lower-temperature thermal regimes. In the thermal regime, recombination of hydrogen nuclei by both free electron capture and charge exchange capture balances ionization, while in the beams the hydrogen 
atom is strictly ionizing and a charge exchange donor. From this viewpoint, the beam atom is distinguished simply as one with a high fixed translational velocity that enters into the collisionality together, possibly, with enhanced state mixing from a motional Stark electric field. Consistent modelling of the type sought here should work smoothly between these different regimes and use the same fundamental data. It is not a difficult task to generalize computationally in this manner and it has the added merit of allowing stringent testing of the Saha-Boltzmann limits and zero-density ionizing extremes. The model summarized in the following sections implements these generalizations, the details of the actual computational implementation are given in section 6 .

\subsection{The bundled-n approach}

Following collisional-radiative theory, the model calculates the quasi-static equilibrium excited population structure relative to the instantaneous hydrogen ground-state and ionizedstate populations in a very many $n$-shell bundled- $n$ approximation. For hydrogen or hydrogenic ions in a plasma, the largest collision cross sections are those for which $n=n^{\prime}$ and $l=l^{\prime} \pm 1$ with ion impact dominating electron impact. For these cases the transition energy is nearly zero and the cross sections are so large that it is a very good approximation to assume relative statistical population for the $l$ states (Burgess and Summers 1974). Thus for hydrogenic systems only populations of complete $n$ shells need be evaluated. Although from an observational spectroscopic point of view, motional Stark separation of low $n$ levels into their Stark manifolds occurs, it remains a very good approximation that relative Stark manifold sub-populations of the same $n$ shell are statistical. The equilibrium populations of the $n$ shells, $N_{n}$, are the solution of the familiar statistical balance equations:

$$
\begin{aligned}
\sum_{n^{\prime}>n}\left[A_{n^{\prime} \rightarrow n}+\right. & \left.N_{\mathrm{e}} q_{n^{\prime} \rightarrow n}^{(\mathrm{e})}+N^{(\mathrm{imp})} q_{n^{\prime} \rightarrow n}^{(\mathrm{imp})}\right] N_{n^{\prime}}+\sum_{n^{\prime \prime}<n}\left[N_{e} q_{n^{\prime \prime} \rightarrow n}^{(\mathrm{e})}+N^{(\mathrm{imp})} q_{n^{\prime \prime} \rightarrow n}^{(\mathrm{imp})}\right] N_{n^{\prime \prime}} \\
& +N_{\mathrm{e}} N_{+} \alpha_{n}^{(\mathrm{r})}+N_{\mathrm{e}}^{2} N_{+} \alpha_{n}^{(3)}+N_{\mathrm{e}} N_{+} \int u(v) B_{\kappa \rightarrow n} \mathrm{~d} \kappa+N_{\mathrm{H}} N_{+} \alpha_{n}^{(\mathrm{cx})} \\
= & \left\{\sum_{n^{\prime}>n}\left[N_{\mathrm{e}} q_{n \rightarrow n^{\prime}}^{(\mathrm{e})}+N^{(\mathrm{imp})} q_{n \rightarrow n^{\prime}}^{(\mathrm{imp})}\right]+\sum_{n^{\prime \prime}<n}\left[A_{n \rightarrow n^{\prime \prime}}+N_{\mathrm{e}} q_{n \rightarrow n^{\prime \prime}}^{(\mathrm{e})}+N^{(\mathrm{imp})} q_{n \rightarrow n^{\prime \prime}}^{(\mathrm{imp})}\right]\right. \\
& \left.+N_{\mathrm{e}} q_{n \rightarrow \epsilon}^{(\mathrm{e})}+N^{(\mathrm{imp})} q_{n \rightarrow \epsilon}^{(\mathrm{imp})}+\int u(v) B_{n \rightarrow \kappa} \mathrm{d} \kappa\right\} N_{n} .
\end{aligned}
$$

$N_{n}$ is the population of the state $n, N_{+}$that of the parent ion (that is, the bare hydrogen nucleus) and $N_{\mathrm{e}}$ is the free-electron density. $N^{\text {(imp) }}$ represents the effective impurity ion density in the plasma. $A_{n \rightarrow n^{\prime}}$ is the usual Einstein coefficient, $q_{n \rightarrow n^{\prime}}^{(\mathrm{e})}$ is the collisional rate coefficient for electrons, $q_{n^{\prime} \rightarrow n^{\prime}}^{(\mathrm{imp})}$ denotes the collective rate coefficient for impurity ions and $q_{n \rightarrow \epsilon}^{(\mathrm{e})}$ and $q_{n \rightarrow \epsilon}^{(\mathrm{imp})}$ are the corresponding coefficients for ionization. $\alpha_{n}^{(\mathrm{r})}, \alpha_{n}^{(3)}$ and $\alpha_{n}^{(\mathrm{cx})}$ are the radiative, three-body and charge exchange recombination rate coefficients respectively. $u(v)$ is the radiation field energy density with $\int u(v) B_{\kappa \rightarrow n} \mathrm{~d} \kappa$ and $\int u(v) B_{n \rightarrow \kappa} \mathrm{d} \kappa$ the induced bound-free photoprocesses. In general several impurity species (nuclei) may be involved in collisionally inducing transitions. Of these the hydrogen nuclei are often the most important. It is helpful to maintain a distinction between the hydrogen nuclei that are the immediate product of ionization of the hydrogen atom population under study (and may feed that population by recombination) from hydrogen nuclei in the plasma, which belong to the impurity ion set. We denote the former as $N_{+}$and the latter as $N_{\mathrm{p}}$ (hereafter we use 'protons' to denote any of the isotopes of hydrogen nuclei in the plasma). Because of better availability of data and approximations for proton induced rates, the protons are sometimes treated as a special (plasma) species and 
the other species treated as the 'impurities'. Let the proton number density be $N_{\mathrm{p}}$ and let the set of impurity charges and fractions by number be $\left\{z_{0_{i}}^{(\mathrm{imp})}, f_{i}^{(\mathrm{imp})} ; i=1, \ldots, I\right\}$. Then the total impurity number density $N^{(\mathrm{imp})}$, effective charge $z_{\text {eff }}$ and component impurity number densities $N_{i}^{(\text {imp) }}$ are related as

$$
\begin{aligned}
& N^{(\mathrm{imp})}=\left(N_{\mathrm{e}}-N_{\mathrm{p}}\right) /\left(\sum_{i=1}^{I} z_{0_{i}}^{(\mathrm{imp})} f_{i}^{(\mathrm{imp})}\right) \\
& z_{\mathrm{eff}}=\left[N_{\mathrm{p}}+N^{(\mathrm{imp})}\left(\sum_{i=1}^{I}\left(z_{0_{i}}^{(\mathrm{imp})}\right)^{2} f_{i}^{(\mathrm{imp})}\right)\right] / N_{\mathrm{e}} \\
& N_{i}^{(\mathrm{imp})}=f_{i}^{(\mathrm{imp})} N^{(\mathrm{imp})} .
\end{aligned}
$$

In the case of a single impurity, which is frequently used as a single 'effective' impurity, it is convenient to alter the definitions. Taking $N_{\mathrm{e}}, N_{\mathrm{p}}$ and $z_{\mathrm{eff}}$ as prescribed

$$
\begin{aligned}
& z_{0}^{(\mathrm{imp})}=\left(z_{\mathrm{eff}} N_{\mathrm{e}}-N_{\mathrm{p}}\right) /\left(N_{\mathrm{e}}-N_{\mathrm{p}}\right) \\
& N^{(\mathrm{imp})}=\left(N_{\mathrm{e}}-N_{\mathrm{p}}\right) / z_{0}^{(\mathrm{imp})}
\end{aligned}
$$

determines the effective charge for the single impurity and its number density.

The population equations are written in terms of Saha-Boltzmann deviation factors, $b_{n}$, Gaunt factors, $g$, and P-factors following Burgess and Summers (1974). The approximate expressions for electron impact rates have to be supplemented by impurity ion impact rates and quadratures altered to allow for the translational motion of the beam atoms. We adopt for baseline ion impact excitation rates the two-state approximation of Vainstein et al (1962) and the semi-empirical formulation of Lodge et al (1976). The primary task to achieve the precision required for beam emission analysis has been the substitution of high-quality collision data from complex collision cross section calculations or experimental cross section measurements for the baseline approximations, see section 5 .

Collectively $q_{n \rightarrow \epsilon}^{\text {(imp) }}$ represents impurity ion impact ionization. This must include both true ion impact ionization and charge transfer so that

$$
q_{n \rightarrow \epsilon}^{(\mathrm{imp})}=q_{\mathrm{ion}, n \rightarrow \epsilon}^{(\mathrm{imp})}+q_{\mathrm{cx}, n \rightarrow s}^{(\mathrm{imp})}
$$

where $s$ is the principal quantum number of the receiver ion. Each of these terms represents a weighted sum over all colliding impurity nuclei. In the present work we have adopted the binary encounter approximation of Percival and Richards (1975) to evaluate cross sections for ion impact ionization from excited states for baseline rates. Again refined fundamental cross section data is used to replace the approximate methods wherever possible.

Although the photo-induced processes have no physical role in the present fusion formulation, we use a diluted (dilution $W$ ) blackbody field to artificially control the ground population and so its influence on higher $n$-shell populations via collisional excitation processes. The last term, $\alpha_{n}^{(\mathrm{cx})}$, denotes state selective charge exchange recombination of $N_{+}$by collision with (plasma) neutral hydrogen. The latter we denote by $N_{\mathrm{H}}$ and it again is to be distinguished from the neutral hydrogen (beam or thermal) whose population is under examination. By appropriate use of $N_{\mathrm{H}}$ and $W$ we can carry out a resolution of effective emission coefficients in terms of the principal driving reactions. These are excitation from the ground state of the atom, free-electron capture and charge exchange recombination from a different neutral hydrogen source. 


\subsection{The derived coefficients}

$S_{\mathrm{cr}}^{(\mathrm{e})}$, is the stopping coefficient for hydrogen beams. If the population equations are written symbolically as

$$
\sum_{n^{\prime}} C_{n, n^{\prime}} N_{n^{\prime}}=r_{n} \quad n=1, \ldots
$$

then

$$
S_{\mathrm{cr}}^{(\mathrm{e})}=1 /\left(N_{\mathrm{e}}\left[C^{-1}\right]_{1,1}\right) .
$$

Note that in conventional practice, this is written in terms of the electron density even though ion collisions are usually the dominating reactants for beam stopping.

Writing the Saha-Boltzmann deviations as $b_{n}$ then $N_{n}=N_{n}^{(\mathrm{S})} b_{n}$, where $N_{n}^{(\mathrm{S})}$ is the SahaBoltzmann population for principal quantum shell $n$, then the switching using $N_{\mathrm{H}}$ and $W$ described earlier allows us to determine the effective collisional-radiative coefficients, $F_{n}^{(1)}$, $F_{n}^{(2)}$ and $F_{n}^{(3)}$ in the expansion of the $b_{n}$ factors as

$$
b_{n}=F_{n}^{(1)} \frac{N_{1}}{N_{+}}+F_{n}^{(2)}+F_{n}^{(3)} \frac{N_{\mathrm{H}}}{N_{\mathrm{e}}} .
$$

The successive terms distinguish the three principal drivers of the population structure. The quantities $F_{n}^{(2)}$ and $F_{n}^{(3)}$ are, respectively, the contributions to the population of level $n$ due to free-electron capture and charge exchange. For a beam atom, $F_{n}^{(1)}$ is the effective contribution to the population of the level $n$ due to excitation processes originating from the ground level. It is the only one of immediate interest for this paper. From it we immediately obtain all effective emission coefficients from the level $n$ as

$$
\epsilon_{n \rightarrow n^{\prime}}=A_{n \rightarrow n^{\prime}}\left(N_{n}^{(\mathrm{S})} / N_{\mathrm{e}} N_{1}\right) F_{n}^{(1)}
$$

where $A_{n \rightarrow n^{\prime}}$ is the Einstein $A$ coefficient. The $F_{n}^{(1)}$ are important in their own right since they give the populations relative to the ground needed for evaluation of excited donor contributions to charge exchange spectroscopy.

For representative JET edge conditions of $N_{\mathrm{e}}=1.0 \times 10^{19} \mathrm{~m}^{-3}$ and $T_{\mathrm{e}}=2000 \mathrm{eV}$ with a pure deuterium plasma and beam energy $4.0 \times 10^{4} \mathrm{eV} / \mathrm{amu}, F_{3}^{(1)}=1.49 \times 10^{9}$ giving $N_{3} / N_{1}=4.96 \times 10^{-4}$. Note that $F_{n}^{(1)}$ is a strongly decreasing function of $n$. The neutral hydrogen number density in the beam is about $10^{16} \mathrm{~m}^{-3}$, the $\mathrm{D}_{\alpha}$ emissivity coefficient $\epsilon_{3 \rightarrow 2}=2.19 \times 10^{15} \mathrm{~m}^{3} \mathrm{~s}^{-1}$ and the stopping coefficient is $1.10 \times 10^{-13} \mathrm{~m}^{3} \mathrm{~s}^{-1}$.

Note also the following implications of the quasi-static equilibrium collisional-radiative assumption under these conditions. The scale length for growth of the $n=3$ hydrogen beam population is about $2.5 \times 10^{-2} \mathrm{~m}$ while the scale length for the $n=3$ population relaxation decreases from about $2.8 \times 10^{-2} \mathrm{~m}$ at the edge of the plasma to about $1.0 \times 10^{-2} \mathrm{~m}$ at the plasma core as collisional depopulation becomes active. Thus the localization of beam emission measurements of the confined plasma, analysed with adoption of the theoretical quasistatic equilibrium assumption, cannot be better than about $3 \times 10^{-2} \mathrm{~m}$ in the JET experiment (see also section 9). Neutral beam diagnostic probes of edge/scrape-off-layer plasma with temperature and density scale lengths of about $3 \times 10^{-2} \mathrm{~m}$ must be modelled in the full, spatially non-equilibrium picture. As has been discussed in previous work, hydrogen with its high first excitation energy is suited as an equilibrium, fast, deep neutral beam probe while a medium/slow lithium beam is suited as a non-equilibrium edge probe. A helium beam, because of the presence of the triplet metastable, shows a mixed character in which the detailed interplay of relaxation times, quasi-static equilibrium assumptions and plasma scale lengths are demonstrated. Analysis of the helium diagnostic beam thus requires generalized collisional-radiative theory and it is the subject of the next paper in this series. 


\section{Fundamental atomic data}

Electron collisions typically contribute less than $20 \%$ to the total collisional rates at JET primary beam energies, although this proportion increases at lower beam energies. This is to be contrasted with the formation of passive Balmer alpha emission from the edge plasma where electron collisions fully dominate. Since the electron speeds are fast compared with beam atom speeds in the beam/plasma overlap region, it is acceptable to draw best available Maxwell averaged effective electron collision strengths from such data collections used for thermal plasmas. These are independent of the beam speed. For the beam atom case, partial effective collision strengths are summed over angular quantum numbers. In the present work, the data of Callaway (1994) were the main original source supplemented by data from Sampson and co-workers (Sampson et al 1983, Golden et al 1981, Clark et al 1982). These data are under revision at the present time to incorporate more precise converged-close coupling (CCC) calculations of Bray and Stelbovics (1992) and Bray (1999) for transitions up to the $n=6$ shell. These data improvements will have important impact on the modelling of the thermal edge plasma, but they are not critical for the present beam case. Electron impact ionization rates are drawn from the compilations of Bell et al (1982).

The principal effort has been in the preparation and updating of comprehensive cross section data for fully ionized impurity ions in collision with neutral hydrogen. The relative speeds of the reactants means that database archives must be maintained as cross sections with explicit Maxwellian averaging carried out in association with the population calculations, as

$$
\left\langle v_{r} \sigma\left(v_{r}\right)\right\rangle=\frac{1}{2} \int_{0}^{\pi} \int_{v_{\min }}^{\infty} v_{r} \sigma\left(v_{r}\right) f\left(v_{r}\right) \sin \theta \mathrm{d} v_{r} \mathrm{~d} \theta
$$

where $\theta$ is defined as the angle between the neutral beam atoms and the plasma ions, which have velocities denoted by $\vec{v}_{\mathrm{p}}$ and $\vec{v}_{t}$ respectively and $v_{r}=\left|\vec{v}_{\mathrm{p}}-\vec{v}_{t}\right|$ is the magnitude of the relative velocity.

For the present work, we have revised and augmented compilations of cross sections for previous JET beam stopping calculations (see von Hellermann and Summers 1993), called the JET89 data to include ion impact ionization and charge exchange data from the ground and excited states up to the $n=5$ shell and ion impact excitation data up to the $n=5$ shell for all bare nucleus impurity species projectiles $\left(\mathrm{H}^{+}-\mathrm{Ne}^{10+}\right)$. The detailed assessment is given by Anderson (1999). Impact ionization from $\mathrm{H}(1 \mathrm{~s})$ has a substantial experimental basis, particularly from the work of Gilbody and co-workers (Shah and Gilbody 1981, Shah et al 1987). More recent comparative assessments are to be found in Janev and Smith (1993) and there are multi-species calculations by Toshima and Tawara (1995). The JET89 data compares well with Janev and Smith with maximum deviations to about $6 \%$ occurring around the cross section peak at about $10^{5} \mathrm{eV} /$ amu for projectiles $\mathrm{H}^{+}-\mathrm{C}^{6+}$ and about $10 \%$ for $\mathrm{O}^{8+}$. Our data for $\mathrm{N}^{7+}$ is interpolated and for $\mathrm{F}^{9+}$ and $\mathrm{Ne}^{10+}$ extrapolated. Our present data, called the JET99 data has been biased to the Janev and Smith values and we anticipate errors $\leqslant 5 \%$. The Toshima and Tawara results are typically about $25 \%$ higher at the cross section peak. Figure 3 is a typical comparison.

For the charge exchange between H(1s) and impurity nuclei, the JET89 data agrees within about $6 \%$ with Janev and Smith and with Toshima and Tawara for $\mathrm{H}^{+}$and $\mathrm{He}^{2+}$ impact, but is discrepant below $10^{4} \mathrm{eV} / \mathrm{amu}$ for the heavier nuclei. This was outside the range of the original JET study and we have taken the opportunity to improve this low-energy region. Figure 4 is typical. The new JET99 data follows Janev and Smith in the low-energy region. Evidently, the variations with our previous data are more substantial as are the results of Toshima and Tawara, so we have a conservative error of about $25 \%$ in the low-energy region. Recent work 


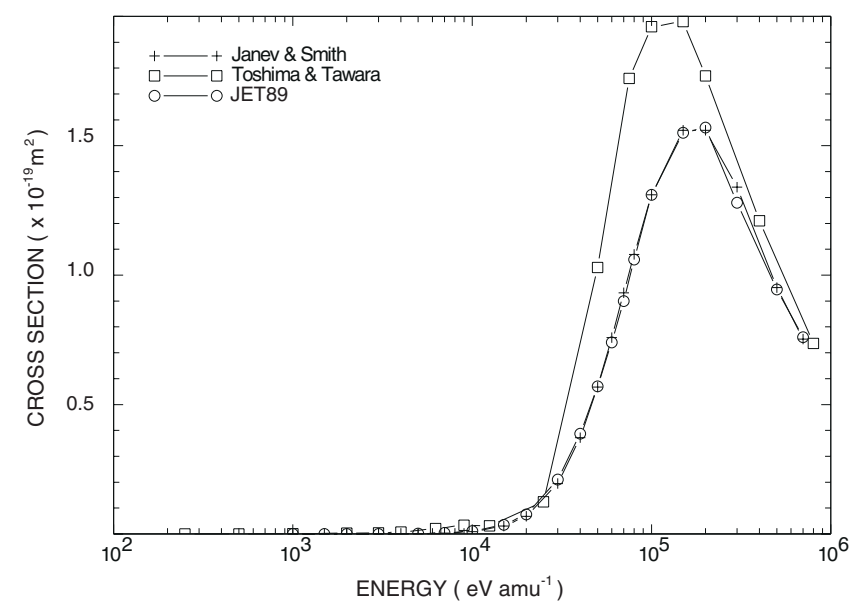

Figure 3. Comparison of ionization cross sections to the final state of the reaction $\mathrm{H}(1 \mathrm{~s})+\mathrm{C}^{6+}$ $\rightarrow \mathrm{H}^{+}+\mathrm{C}^{6+}$.

by Busnengo et al (1996) supports the cross section estimates in the higher-energy region typical of the JET primary beam energies.

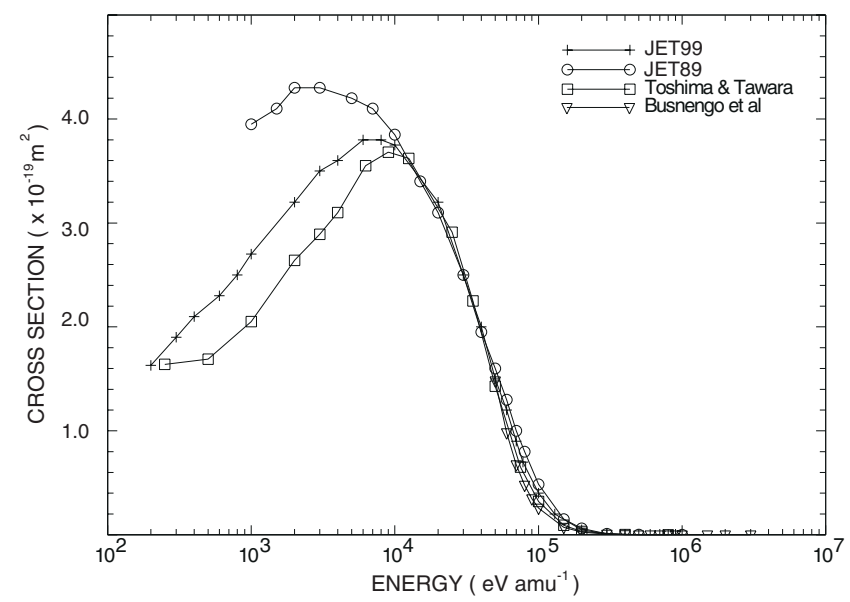

Figure 4. Comparison of charge exchange cross sections for the reaction $\mathrm{H}(1 \mathrm{~s})+\mathrm{B}^{5+} \rightarrow \mathrm{H}^{+}+\mathrm{B}^{4+}$.

For ion impact excitation from $\mathrm{H}(n=1)$ to $\mathrm{H}(n=2)$ by $\mathrm{H}^{+}$and the light ion bare nuclei, recent work (Janev and Krystic 1992, Ermolaev 1990, Fritsch and Lin 1991, Toshima and Tawara 1995) together with the appraisal by Janev and Smith (1993) indicated only modest adjustments to the JET89 data of at most $10 \%$. For the key excitations from $\mathrm{H}(n=1)$ to $\mathrm{H}(n=3)$, adjustments by at most $15 \%$ over the whole energy range were required, except for $\mathrm{H}^{+}$impact at energies below $10^{4} \mathrm{eV} / \mathrm{amu}$ where the new JET99 data follows Janev and Smith. Thus the uncertainty in the JET99 data is $\leqslant 10 \%$. For excitations $\mathrm{H}(n=1)$ to $\mathrm{H}(n=4)$ and $\mathrm{H}(n=5)$ the JET89 data was based on scaling. Comparison is possible with Toshima and Tawara up to $\mathrm{H}(n=4)$ and all light nuclei projectiles up to $\mathrm{O}^{8+}$ and with Janev and Smith for $\mathrm{He}^{2+}$. The Toshima and Tawara close coupling calculations terminated with the $n=4$ shell and consequently their results are clearly too large, exceeding in many cases the cross sections 


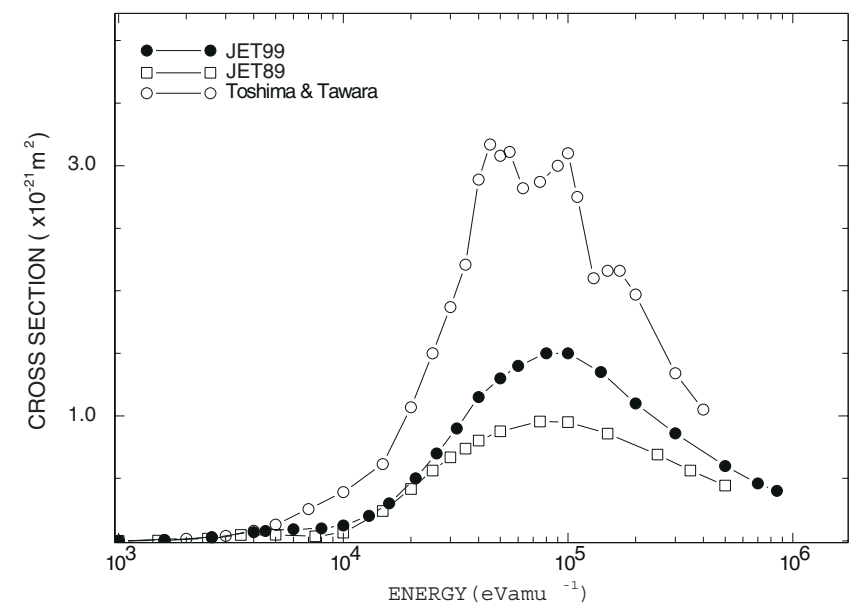

Figure 5. Comparison of excitation cross section for the reaction $\mathrm{H}(n=1)+\mathrm{He}^{2+} \rightarrow$ $\mathrm{H}(n=4)+\mathrm{He}^{2+}$
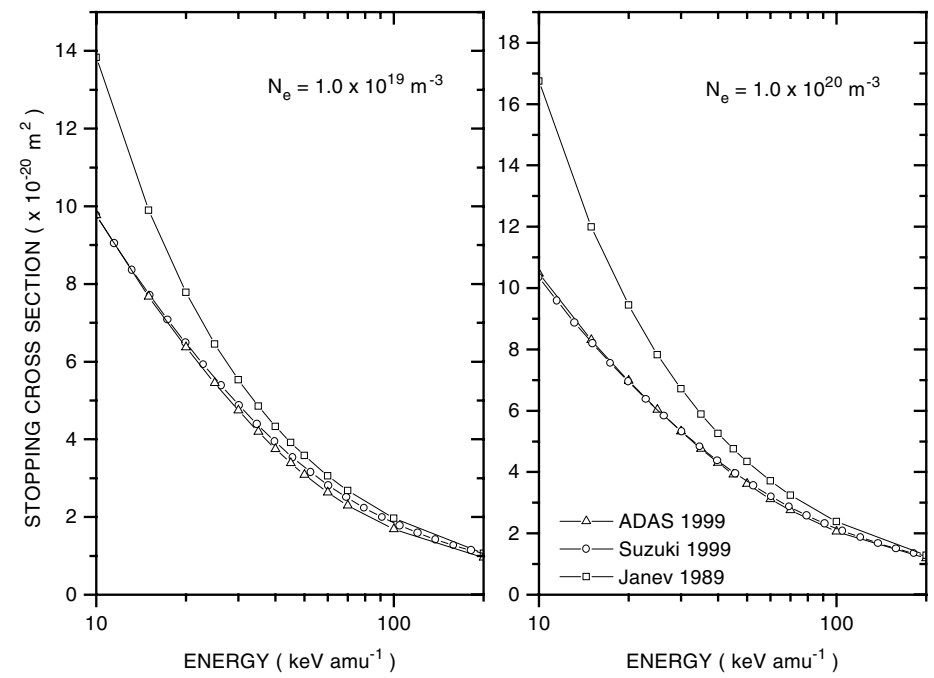

Figure 6. Comparison of stopping cross sections for a pure $\mathrm{H}^{+}$plasma at $1.0 \times 10^{19}$ and $1.0 \times 10^{20} \mathrm{~m}^{-3}$. In both cases the electron temperature $T_{\mathrm{e}}(\mathrm{keV})$ and the ion temperature $T_{\mathrm{i}}(\mathrm{keV})$ are taken to be $E(\mathrm{keV} / \mathrm{amu}) / 10$.

to $n=3$. The Janev and Smith preferred cross section for $\mathrm{He}^{2+}$ impact confirms this, but their data is about $40 \%$ larger than JET89 at the cross section peak and is to be preferred (see figure 5). For projectiles $\mathrm{Li}^{3+}$ to $\mathrm{Ne}^{10+}$, the JET99 data continues to use scaled values, but the uncertainty cannot be less than 50\%. For ion impact excitation from excited $\mathrm{H}$ states there are no high-quality data and the JET99 scaled data uncertainties must be up to $100 \%$.

For ionization from excited states comparison is possible with Janev and Smith for $\mathrm{H}^{+}$ impact. The JET89 data are lower by up to 50\% with a marked shift in the cross section peak to lower energies evident for $\mathrm{H}(n \geqslant 4)$. The JET99 data have been revised in the light of this with an uncertainty not less that 30\%. For impact by impurity nuclei, JET99 continues to use scaled data. These remain the least known processes with an uncertainty not less than 
$100 \%$. For charge transfer from excited states, the ADAS database has extensive high-quality data for transfer from $\mathrm{H}(n=2)$ for all light nuclei projectiles and this is scaled for higher $n$ shells. Separate studies of such cross sections associated with charge exchange spectroscopy (Hoekstra et al 1998) give us some confidence in the scaling here. In summary, excitation and ionization from excited states are the fundamental data with the greatest uncertainties, although the consequential error for the effective beam stopping and beam emission coefficients is the key issue for the present diagnostic analysis (see section 7). Figure 6 compares the beam stopping cross sections obtained using the JET99 data (ADAS 1999) with the work of Suzuki et al (1998) and Janev et al (1989) (the stopping cross section being defined as the ratio of the effective beam stopping coefficient to the beam speed). As discussed by Suzuki et al (1998), differences in the cross sections employed to describe electron loss from the $\mathrm{H}(n=1)$ ground state by Janev et al (1989) give rise to an increase in the stopping cross section in the low-energy regime.

\section{Computational implementation}

The computer codes associated with the present work have been in development over a period of nearly 15 years. Also, key derived data from the codes have been distributed to many fusion laboratories throughout the world, which created a need at JET to systematize the work and establish appropriate practice for maintenance, update and revision of the codes and data. The result was an integrated atomic data and modelling system at JET called the 'Atomic Data and Analysis Structure', ADAS (Summers, 1994). It has now been extended to the international ADAS Project (Summers, 1999) of which the beam stopping and emission modelling and data form part. Within ADAS many data classes (called ADAS data formats -adf's) are required and we have given attention to prescribing the precise content and organization of each data format so that the derived data fully satisfies the need of the fusion application and fundamental data formats are suitable for direct creation by calculators of source cross section data. Two ADAS codes and six data formats provide the computational and data archiving functionality for the beam analysis, as shown in the schematic diagram, figure 7 . The fundamental data formats are adf02 - fundamental ion-atom collision cross section data; adf04-fundamental electron collisional rate coefficient data and $A$ values; and adf07-fundamental electron impact ionization collision data. The derived data formats for applications are adf21-effective beam stopping coefficients; adf22-effective $\mathrm{D}_{\alpha}$ beam emission coefficients; and adf26collisional-radiative bundled- $n$ population tabulations.

The program ADAS310 is a full implementation of the collisional-radiative model for hydrogen in the bundled- $n$ approximation as discussed earlier. Typically, about $100 n$ shells are included and condensation techniques are employed with about 25 representative $n$ shells selected (Burgess and Summers 1974). It is driven by an IDL graphical user interface with the actual calculations carried out using FORTRAN routines which are transparent to the user. ADAS310 iterates through sets of plasma conditions, which include the plasma density, temperature and neutral beam energy. For each set of conditions, a subset of cases are executed by switching on and off the three primary drivers so that the effective contributions to the excited state populations of the atom in the collisional-radiative sense by each primary driver separately can be isolated. This is achieved in the code by alteration of the neutral hydrogen density $N_{\mathrm{H}}$ and the photoionizing radiation field dilution for the ground state. Solution is carried out in the $c_{n}\left(b_{n}-1\right)$ and $\exp \left(I_{n}\right) b_{n}$ representations with a switch between the two at $n=5$ to avoid cancellation error and the solution presented in the more usual $b_{n}$ representation. Output from ADAS310 includes tabulations of the $F$ 's, $N^{(\mathrm{S})}$ 's and the collisional-radiative ionization and recombination coefficients $S_{\mathrm{cr}}$ and $\alpha_{\mathrm{cr}}$, with and without the influence of charge 


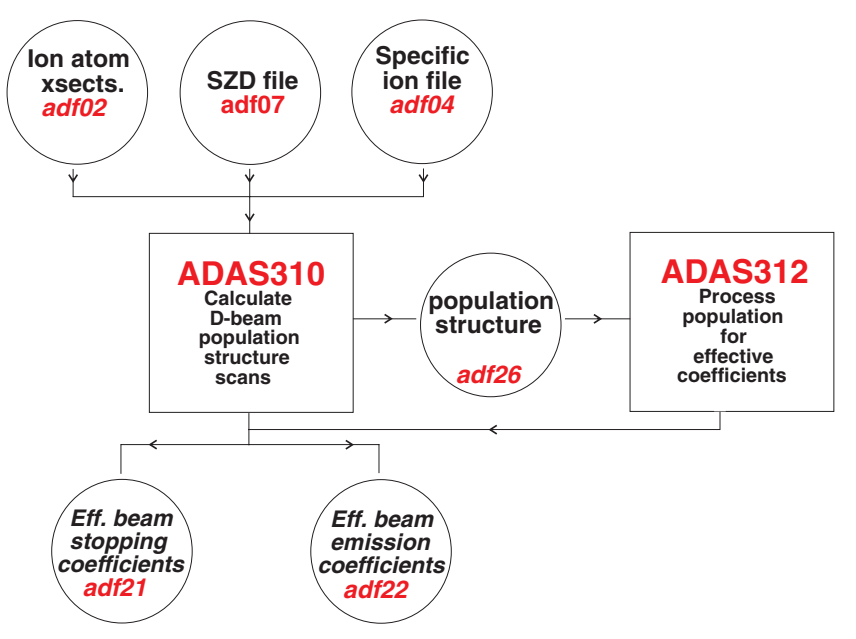

Figure 7. Schematic overview of the production of the derived coefficients. The ADAS adf02, adf04 and adf07 data files are structured assemblies of fundamental reaction cross sections and rates. The adf $21, \operatorname{adf} 22$ and adf 26 data files contain derived data generated by the collisional radiative codes.

transfer, at each set of plasma conditions. This is a structured output organized according to ADAS data format, adf26. The program ADAS310 is too slow in execution for a direct link to inter-pulse experiment analysis at JET and so it is necessary to prepare compact tabulations of effective beam stopping and emission coefficients for subsequent look-up by CHEAP.

ADAS312 is the interactive post-processing code designed to extract effective stopping and emission coefficients from the comprehensive adf 26 files and archive the data in condensed rapid-look-up tables in their respective ADAS data formats of adf 21 and adf 22 . The effective coefficients are most sensitive to the beam particle energy and the plasma ion density and less sensitive to plasma ion temperature. Suitable tabulations can therefore be built on a reference set of plasma and beam conditions, namely a two-dimensional array of coefficients as functions of beam energy and plasma density at the reference conditions of the plasma ion temperature and then a one-dimensional vector of coefficients as a function of the plasma ion temperature at the reference conditions of the other parameters. ADAS310 accepts as input the definitions of these scans, establishes an extended list of cases required to achieve the latter and then executes repeated population calculations at each set of plasma conditions in the list.

ADAS310 can compute the populations for any mixture of light impurities $\left(\mathrm{H}^{+}\right.$to $\left.\mathrm{Ne}^{10+}\right)$ in the plasma. It is impractical to tabulate all possible mixtures of impurities. It is our usual practice to execute ADAS310 in turn for each light impurity species from hydrogen to neon treated as a pure species. The mixed species effective coefficients are constructed from these pure impurity solutions as a linear superposition by the theoretical data acquisition routines in CHEAP. Thus the composite collisional radiative stopping coefficient is

$S_{\mathrm{cr}}\left(E_{\mathrm{B}}, N_{\mathrm{e}}, T_{\mathrm{I}}\right)=\sum_{i=1}^{I}\left[z_{0_{i}}^{(\mathrm{imp})} f_{i}^{(\mathrm{imp})} S_{\mathrm{cr}}^{(i)}\left(E_{b}, N_{\mathrm{e}}^{(i), \text { equiv }}, T_{I}\right)\right] /\left(\sum_{i=1}^{I}\left[z_{0_{i}}^{(\mathrm{imp})} f_{i}^{(\mathrm{imp})}\right]\right)$

where $f_{i}^{(\mathrm{imp})}$ is the fraction of impurity $i . N_{\mathrm{e}}^{(i) \text {, equiv }}$ is the equivalent electron density to be used in the extraction of the stopping coefficient contribution from the $i$ th pure impurity archive 


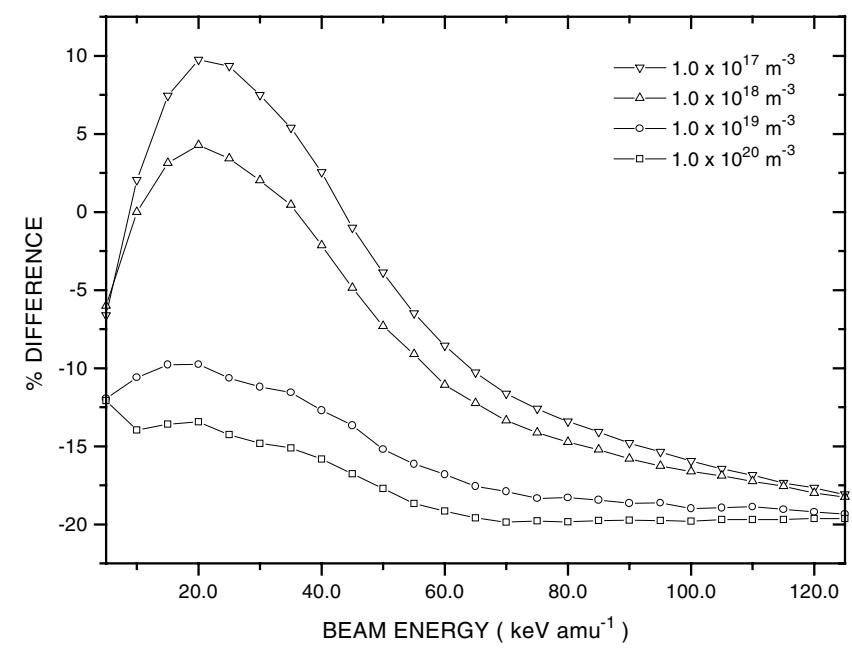

Figure 8. Percentage difference between the exact results from ADAS310 and the linear combination/interpolation from the fast-look-up tables. The composite plasma consists of $70 \%$ $\mathrm{D}^{+}, 20 \% \mathrm{C}^{6+}$ and $10 \% \mathrm{Be}^{4+}$. The reference values are $E_{\mathrm{B}}^{(\mathrm{ref})}=40.0 \mathrm{keV} / \mathrm{amu}, N_{\mathrm{e}}^{(\mathrm{ref})}=$ $6.78 \times 10^{17} \mathrm{~m}^{-3}$ and $T_{\mathrm{I}}^{(\mathrm{ref})}=2.0 \times 10^{3} \mathrm{eV}$.

and is given by

$$
N_{\mathrm{e}}^{(i) \text {,equiv }}=\left(N_{\mathrm{e}} / \sum_{k=1}^{I}\left[z_{0_{k}}^{(\mathrm{imp})} f_{k}^{(\mathrm{imp})}\right]\right)\left(\sum_{k=1}^{I}\left[\left(z_{0_{k}}^{(\mathrm{imp})}\right)^{2} f_{k}^{(\mathrm{imp})}\right]\right) / z_{0_{i}}^{(\mathrm{imp})} .
$$

The coefficient is written in terms of the primary parameters $E_{\mathrm{B}}$, the beam energy, $N_{\mathrm{e}}$ and $T_{\mathrm{I}}$, the impurity ion temperature, assumed to be the same for all impurities.

The same approximations and compact tabulation organizations are used for both beam stopping and beam emission coefficients. A detailed error analysis of the effect of these two approximations has been carried out by Anderson (1999) by comparison with ADAS310 calculations using the true mixtures. The linearly superposed beam stopping coefficient for a plasma of $70 \% \mathrm{D}^{+}, 20 \% \mathrm{C}^{6+}$ and $10 \% \mathrm{Be}^{4+}$ is in error by at most $2 \%$ for reference conditions $E_{\mathrm{B}}^{(\mathrm{ref})}=4.0 \times 10^{4} \mathrm{eV} / \mathrm{amu}, N_{\mathrm{e}}^{(\mathrm{ref})}=10^{19} \mathrm{~m}^{-3}$ and $T_{\mathrm{I}}^{(\mathrm{ref})}=2.0 \times 10^{3} \mathrm{eV}$ for $10^{4} \mathrm{ev} / \mathrm{amu} \leqslant E_{\mathrm{B}} \leqslant 10^{5} \mathrm{eV} / \mathrm{amu}$ and $10^{16} \mathrm{~m}^{-3} \leqslant N_{\mathrm{e}} \leqslant 10^{20} \mathrm{~m}^{-3}$ while interpolation in ion temperature contributes a further $1 \%$ in the range $10^{2} \mathrm{eV} \leqslant T_{\mathrm{I}} \leqslant 5 \times 10^{3} \mathrm{eV}$. The errors from the compact tabulations of the beam emission coefficient are more substantial, but less than $15 \%$ for reasonable ranges of plasma and beam conditions. Figure 8 shows an extreme case where the reference electron density is set an unrealistic low value. From this evidence, the design of the fast-look-up tables preserves the accuracy level required for analysis of both calculated and measured beam densities, but it is appropriate to re-tune the tabulations to reference beam and plasma conditions for application to other plasma/injection systems.

\section{Behaviours and sensitivities of the effective coefficients}

The parameters to which the effective stopping and emission coefficients are most sensitive are the electron density (note again that impurity ion collisions dominate at heating beam energies but coefficients are expressed in terms of electron density) and the neutral beam energy, with weaker dependence on the ion temperature and the nuclear charge of the fully stripped plasma 
impurity ions. The general behaviour of the effective coefficients is quite well known, so in this section only a brief overview is given for completeness. The main emphasis of this section is on the influence of uncertainties in the fundamental atomic data on the effective coefficients.

\subsection{The effective stopping coefficient}

Figure 9 shows the energy and density dependence of the effective stopping coefficient for both a pure $\mathrm{D}^{+}$plasma and a pure $\mathrm{C}^{6+}$ plasma. The coefficients exhibit the familiar low- ('coronal picture') and high-density asymptotic limits. The departure from the coronal limit appears around approximately $10^{16} \mathrm{~m}^{-3}$, while the formation of the high-density limit is approached at about $10^{24} \mathrm{~m}^{-3}$. The behaviour with increasing neutral beam energy reflects the change from charge exchange as a loss mechanism to ion impact ionization. The behaviours for the pure $\mathrm{D}^{+}$plasma and pure $\mathrm{C}^{6+}$ plasma are qualitatively similar, but the $\mathrm{C}^{6+}$ plasma ions are somewhat more efficient at stripping the electrons from the beam atoms at higher energies. The resolution of the figure at low beam energies is insufficient to show the transition to electron collisions as the main stopping mechanism. Figure 10 shows the weak temperature dependence of the stopping coefficient for a pure $\mathrm{D}^{+}$plasma. Figure 11 shows the effective stopping coefficient as a function of beam energy for a variety of pure impurity plasmas. The impurity ion nuclear charge dependence in the low-energy region is due to the dominating charge exchange collisions which scale approximately with the nuclear charge of the receiver ion. Since the electron density is fixed and charge neutrality imposed, this appears as a weak charge dependence. A near coincidence of curves in the beam range of current tokamaks can be observed. The charge dependence of the effective stopping coefficient in the high-energy region comes from the ion impact ionization cross sections which scale approximately as the impurity nuclear charge squared.

The accuracy at which the effective stopping coefficient can be calculated is governed by the accuracy at which the fundamental data is known. It is therefore of interest to investigate the influence of the uncertainty associated with the fundamental data on the behaviour of the effective stopping coefficient. In figure 12, working from left to right, we show the influence of individually increasing the cross sections for ion impact excitation from the ground state to the $n=2,3$ and 4 quantum shells by $20 \%$. Increasing the cross section data for excitation only becomes important when the electron density is sufficiently high. In such circumstances modifying the data for the $n=1 \rightarrow 2$ transition has the most significant effect. Due to the large cross section for ion impact ionization from the $n=2$ shell, when the $n=2$ shell population is enhanced, the rate at which electrons are stripped from the beam atoms increases. We now consider modifying the data for ion impact excitation associated with the excited states of the beam atoms. Figure 13 shows the influence of collectively increasing the cross sections by $100 \%$ for the transitions between the quantum shells $n \rightarrow n^{\prime}$, where $n \in[2,3]$ and $n^{\prime} \in[3,5]$ $\left(n \neq n^{\prime}\right)$. A maximum difference of about $4.0 \%$ can be observed. Similar studies have been undertaken for the direct atomic processes associated with the ground and excited states. Increasing the data by $10 \%$ for both charge exchange and ion impact ionization from the ground state results in a typical increase in the stopping coefficient of about $5.0 \%$, while modifying the cross section data associated with the excited states by $50.0 \%$ only gives rise to an increase in the stopping coefficient of around $2.0 \%$.

\subsection{The $D_{\alpha}$ effective emission coefficients}

Figure 14 shows the energy and density dependence of the $\mathrm{D}_{\alpha}$ beam emission coefficient for both a pure $\mathrm{D}^{+}$plasma and a pure $\mathrm{C}^{6+}$ plasma. The coronal limit now corresponds to the 

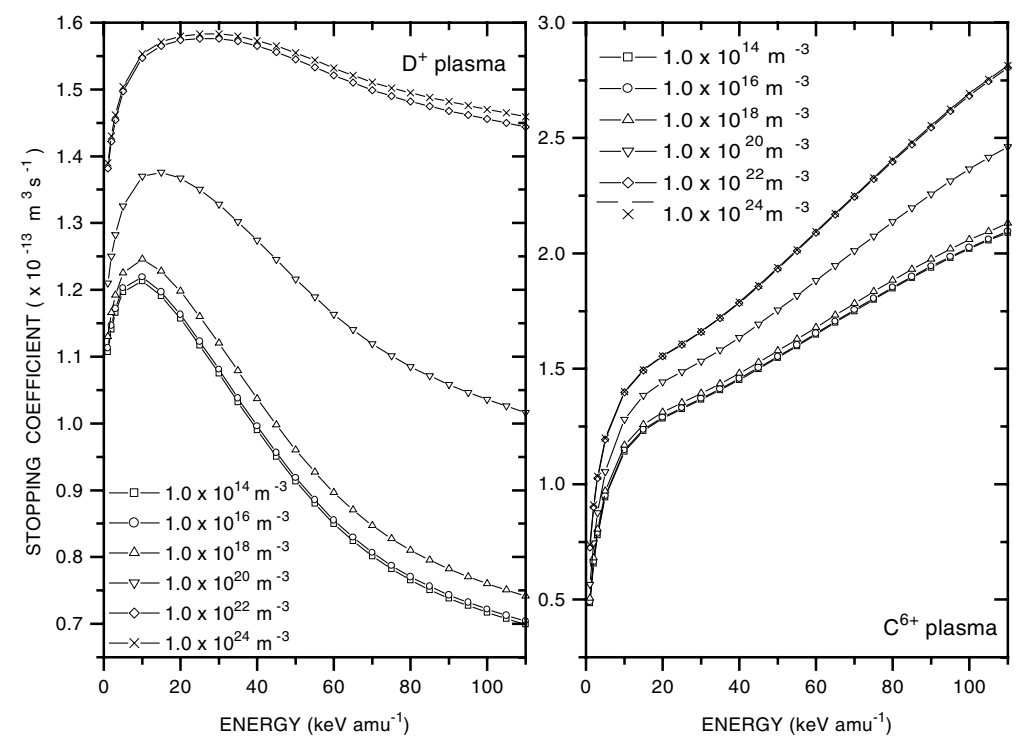

Figure 9. Energy and density dependence of the effective stopping coefficient. $T_{\mathrm{i}}=2.0 \times 10^{3} \mathrm{eV}$ The figure on the left is for a pure $\mathrm{D}^{+}$plasma and on the right is for a pure $\mathrm{C}^{6+}$ plasma. Characteristic are the low- and high-density asymptotic limits and the change from charge transfer to ionization as the dominant loss mechanism at higher energies. The slope variation at $E_{\mathrm{B}}=30 \mathrm{keV} / \mathrm{amu}$ in the $\mathrm{C}^{6+}$ plasma shows this most clearly.

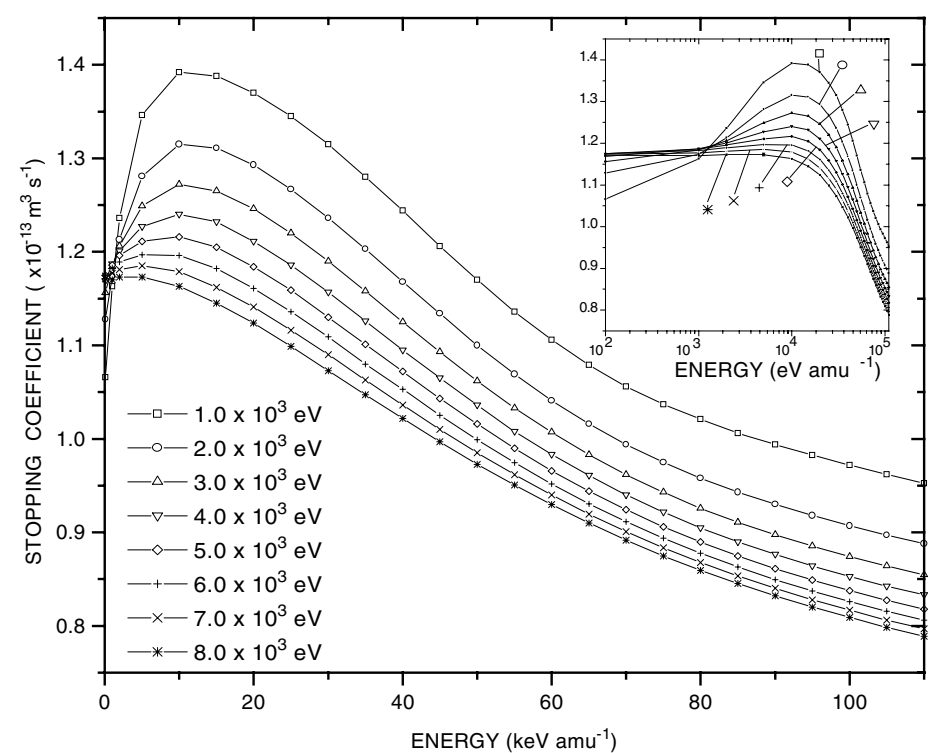

Figure 10. Temperature dependence of the effective beam stopping coefficient for a pure $\mathrm{D}^{+}$ plasma. $N_{\mathrm{e}}=3.0 \times 10^{16} \mathrm{~m}^{-3}$.

largest values of the effective coefficient, which is progressively reduced at higher electron densities by ionization losses (direct and indirect) out of the $n=3$ level. The energy scale is 


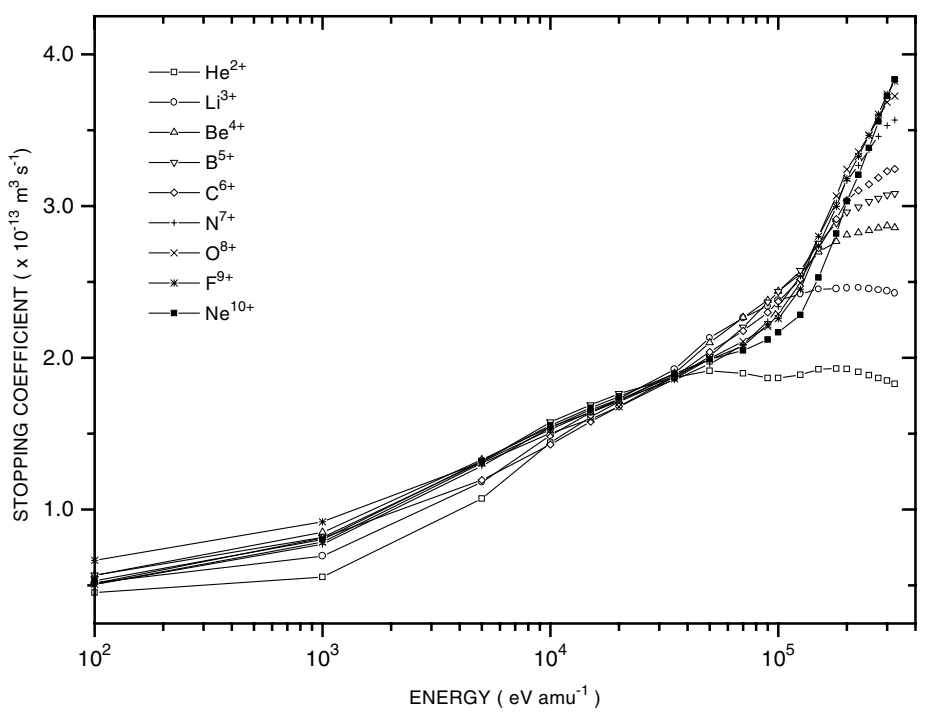

Figure 11. Beam stopping coefficient for a range of pure impurity plasmas. $N_{\mathrm{e}}=3.0 \times 10^{19} \mathrm{~m}^{-3}$ with charge neutrality imposed. $T_{\mathrm{i}}=2.0 \times 10^{3} \mathrm{eV}$.

logarithmic and shows the electron collision driven region at low energy $\left(E_{\mathrm{B}} \leqslant 10^{4} \mathrm{eV} / \mathrm{amu}\right)$. As the beam energy increases the ion collisions become important and the results from the two types of plasma differ more substantially. Figure 15 shows the temperature dependence of the $\mathrm{D}_{\alpha}$ effective emission coefficient. The influence of the nuclear charge of the plasma impurity ions is shown in figure 16 for a range of pure impurity plasmas. There is a small thermal contribution from each ion which gives rise to the nuclear charge dependence of the emission coefficient in the low-energy regime.

We now consider the influence of the uncertainties associated with the fundamental atomic data on the $\mathrm{D}_{\alpha}$ emission coefficient. In figure 17, working from left to right, we show the results of individually increasing the cross section data for ion impact excitation from the ground state to the $n=2,3$ and 4 quantum shells by $20 \%$. Modifying the collisional excitation cross section for the $n=1 \rightarrow 3$ transition has the greatest influence on the $\mathrm{D}_{\alpha}$ emission coefficient. It is worth noting that the modified data for the $n=1 \rightarrow 3$ transition had little effect on the stopping coefficient. Figure 18 shows the influence of the uncertainties in the fundamental data for the atomic processes associated with the excited states. The plot on the left in figure 18 illustrates the results of increasing the cross sections for charge exchange and ion impact ionization associated with the $n=2,3$ and 4 quantum shells by 50\%. As the electron density increases, the role of stepwise atomic process becomes important. At a density of $3.0 \times 10^{19} \mathrm{~m}^{-3}$ a maximum difference of around $20.0 \%$ can be observed as a result of the modified data. The plot on the right in figure 18 exhibits the influence of collectively increasing the excitation cross sections by $100 \%$ for the transitions $n \rightarrow n^{\prime}$, where $n \in[2,3]$ and $n^{\prime} \in[3,5]$. The influence of stepwise atomic processes can again be observed as the electron density is increased and at $3.0 \times 10^{19} \mathrm{~m}^{-3}$ the influence of the modified data on the emission coefficient is about $22.0 \%$. We have also considered the implications of increasing the cross section data for the direct processes associated with the ground state. Modifying the data for both charge exchange and ion impact ionization has a negligible effect on the $\mathrm{D}_{\alpha}$ emission coefficient. 

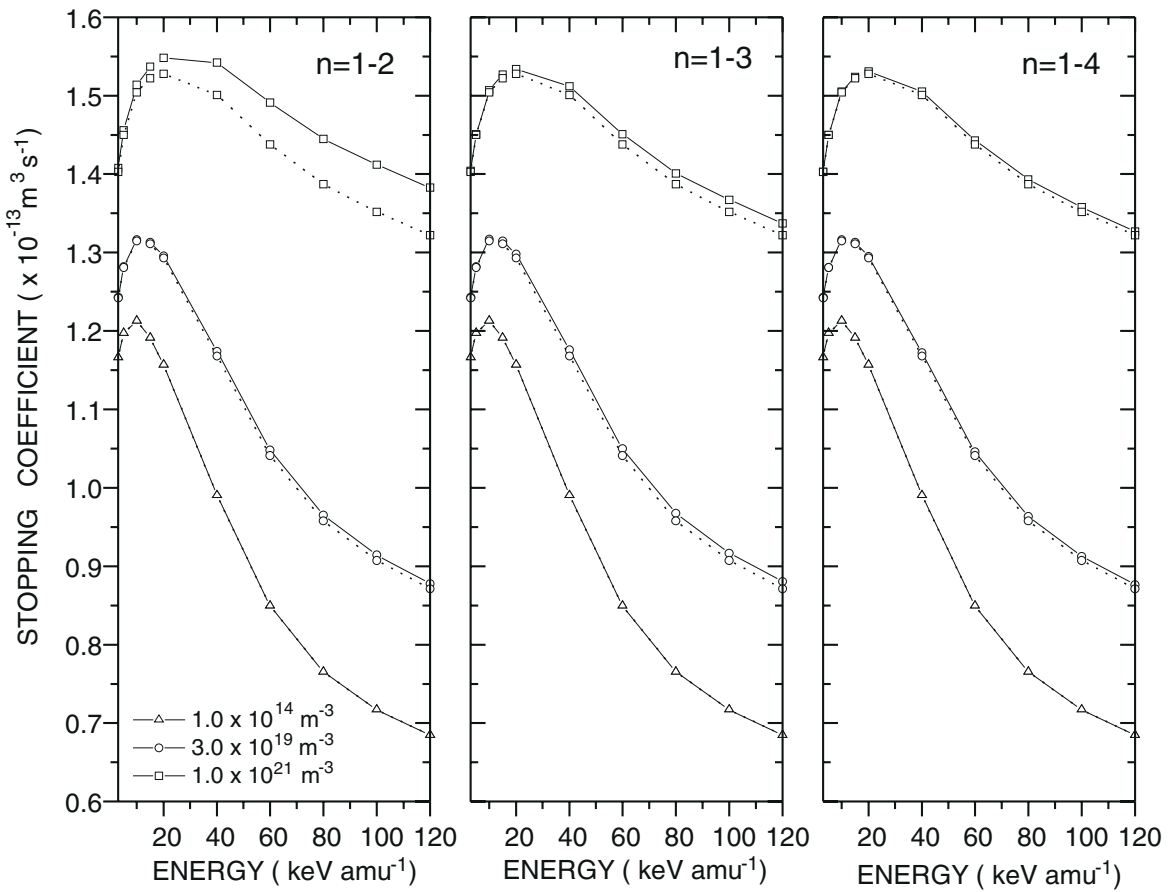

Figure 12. Influence of the fundamental data on the effective stopping coefficient for a pure $\mathrm{D}^{+}$ plasma. The ion temperature was $2.0 \times 10^{3} \mathrm{eV}$. The broken curves represent the results from the unmodified database. The full curves are the results obtained by modifying the respective ion impact excitation cross sections by $20 \%$.

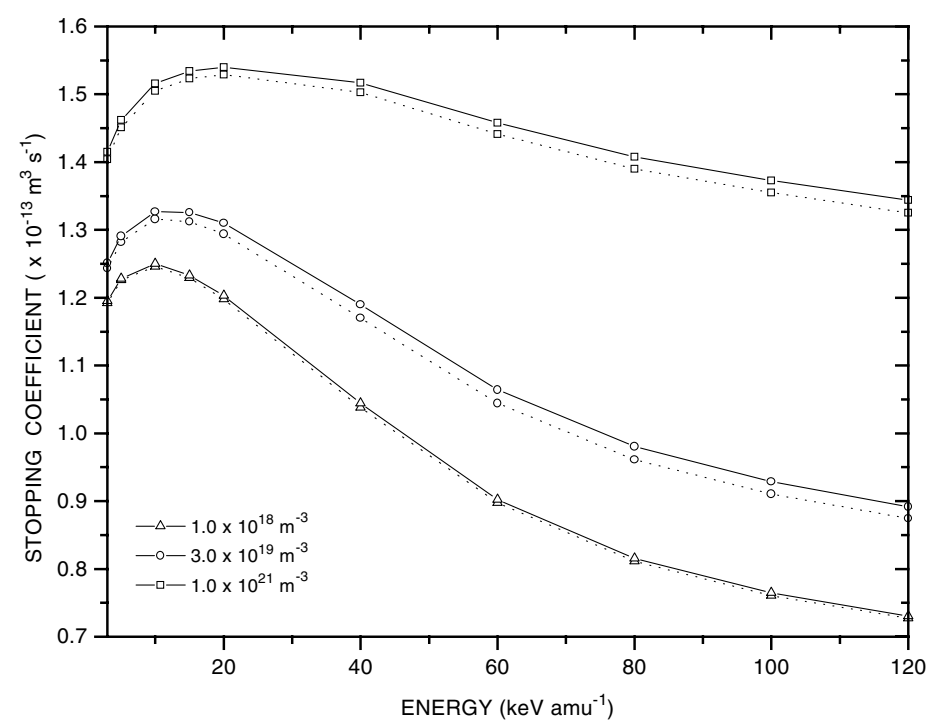

Figure 13. Behaviour of the effective stopping coefficient for a pure $\mathrm{D}^{+}$plasma. The broken curves are the results from the unmodified database. The full curves are the results obtained from collectively increasing the excitation cross sections by $100 \%$ for the transitions $n \rightarrow n^{\prime}$, where $n \in[2,3]$ and $n^{\prime} \in[3,5]$. 


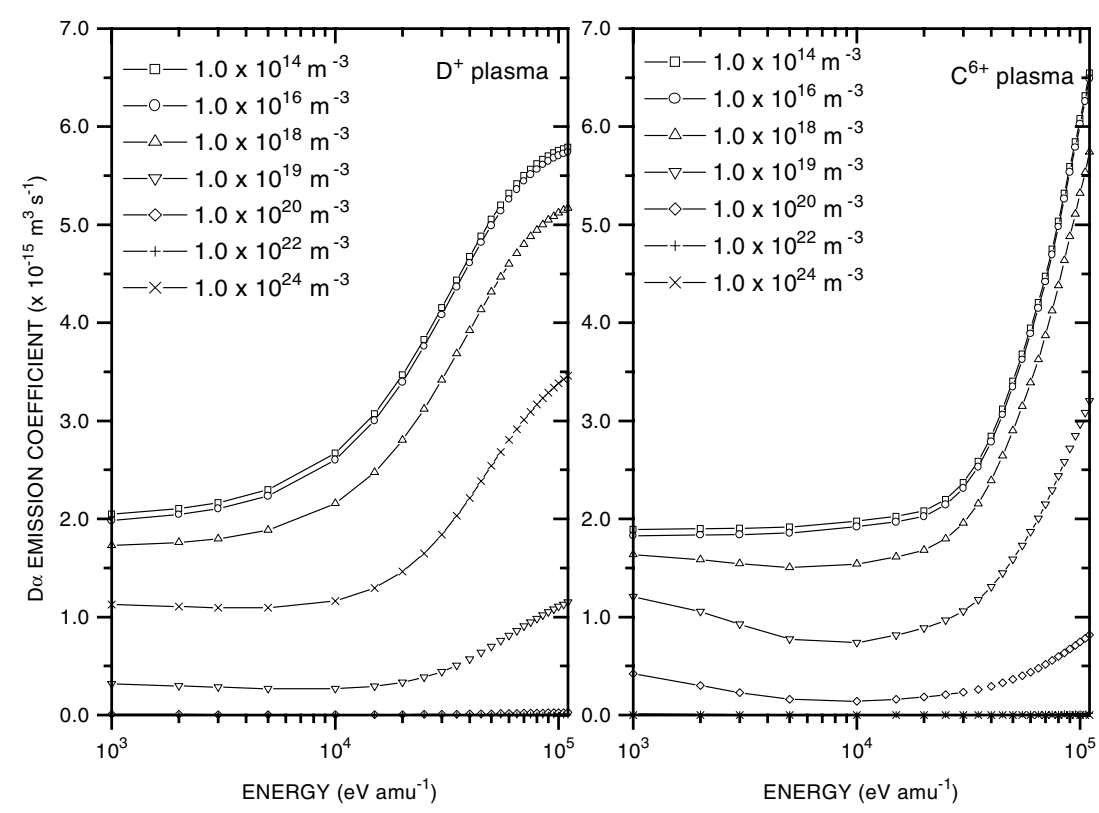

Figure 14. Energy and density dependence of the $\mathrm{D}_{\alpha}$ effective emission coefficient. The figure on the left is for a pure $\mathrm{D}^{+}$plasma and that on the right for a pure $\mathrm{C}^{6+}$ plasma. $T_{\mathrm{i}}=2.0 \times 10^{3} \mathrm{eV}$.

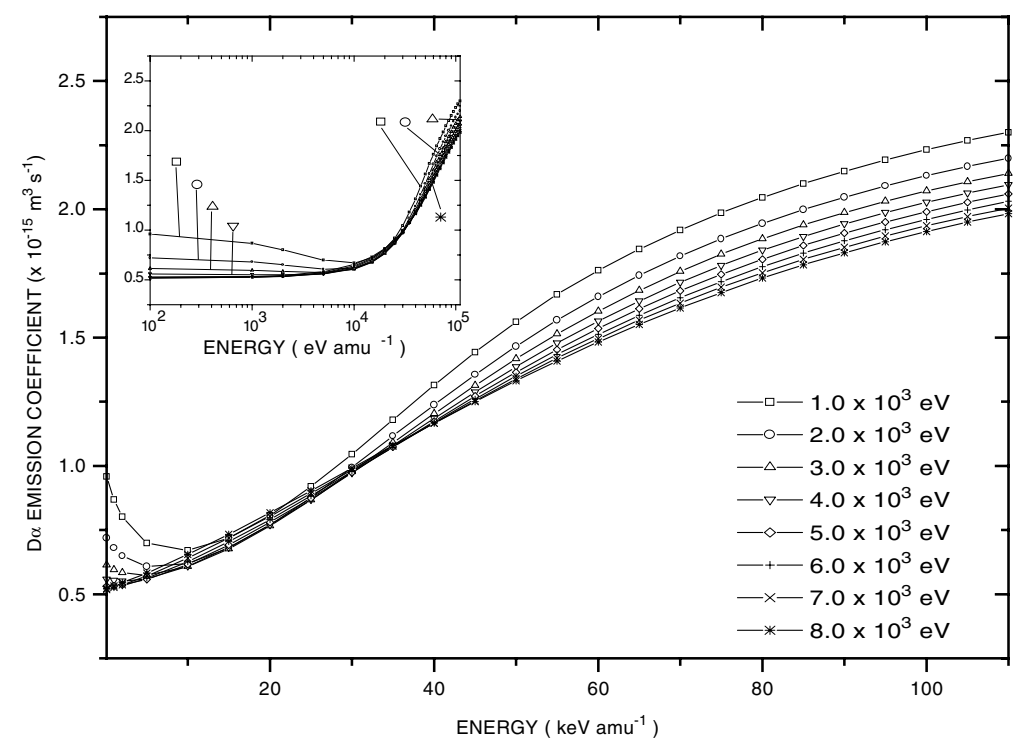

Figure 15. Effective $\mathrm{D}_{\alpha}$ emission coefficient for a pure $\mathrm{D}^{+}$plasma. $N_{\mathrm{e}}=3.0 \times 10^{19} \mathrm{~m}^{-3}$ and $T_{\mathrm{i}}=2.0 \times 10^{3} \mathrm{eV}$

\section{Results}

Due to the modifications required to enable the spectral analysis code to address double beam bank spectra, it was necessary to validate its operation and the subsequent integration of the 


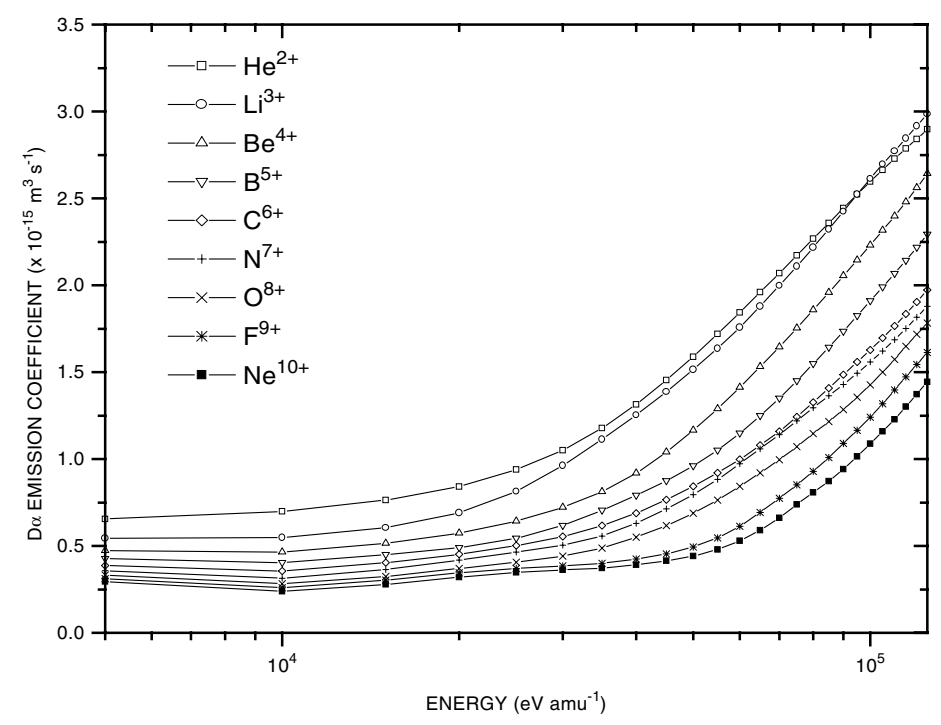

Figure 16. Effective $\mathrm{D}_{\alpha}$ emission coefficient for a range of pure impurity plasmas. $T_{\mathrm{i}}=2.0 \times 10^{3} \mathrm{eV}$ and $N_{\mathrm{e}}=3.0 \times 10^{19} \mathrm{~m}^{-3}$ with the condition of charge neutrality imposed.

measured beam emission flux into the CHEAP package. The method adopted in the present work was to analyse spectra associated with single beam bank pulses, since such analysis had previously been undertaken by Mandl et al (1993). After which we considered the analysis of double beam bank spectra, which are new. We have also taken the opportunity to examine the pulse-to-pulse variation between the measured and calculated line integrated beam densities for both single and double beam bank pulses.

It should be noted that the duration for which the neutral beams are active can vary between pulses, under normal operating conditions a period of $10 \mathrm{~s}$ is typical. In such circumstances this allows at least 200 spectra in total being recorded at each radial position, thus providing an abundance of both radial and time-dependent measurements of the beam emission flux. Figure 19 shows the radial and time dependence of the measured line integrated beam density obtained during the single beam bank pulse No 40419. The neutral beam density obtained from the numerical attenuation calculation is also shown for comparison. A characteristic feature observed from the analysis of all single beam bank spectra is the fall-off of the measured beam density near the edge of the plasma $(\sim 3.9 \mathrm{~m})$. This was first observed by Mandl et al (1993) and still remains an unresolved problem. Nevertheless, the agreement shown in figure 19 is representative of the typical results obtained.

Motivated by the temporal variation of C II passive emission lines which would occasionally pollute the beam emission spectrum (Anderson 1999), it was our intention to study the pulse-to-pulse variability of the results obtained for L- and H-mode plasmas (Wagner et al 1982). The latter plasma regime reduces the presence of partially ionized species between the last-closed magnetic flux surface and the edge of the vessel, minimizing the polluting passive emission. However, due to the lack of experimental data our selection criteria was very simple, essentially a pulse was selected for analysis provided that neutral beam injection was continuous and did not exhibit any anomalous behaviour. Figure 20 shows the variation between the measured and calculated line integrated beam densities for a range of single beam bank pulses. Each data point represents a time averaged value for a fixed radial position. The 

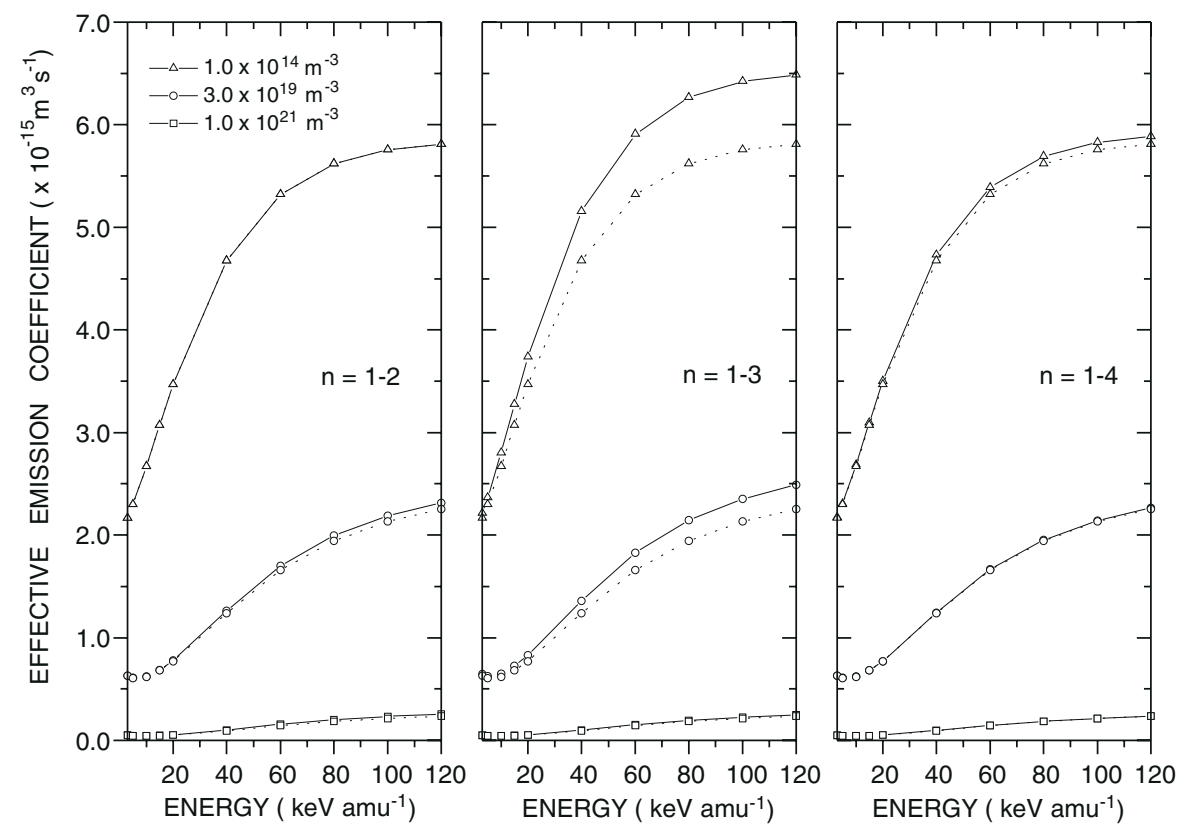

Figure 17. Effective $\mathrm{D}_{\alpha}$ emission coefficient for a pure $\mathrm{D}^{+}$plasma. Working from left to right are the results of increasing the excitation cross sections from the ground to the $n=2,3$ and 4 shells by $20 \%$. The broken curves show the results obtained from the unmodified data. $T_{\mathrm{i}}=2.0 \times 10^{3} \mathrm{eV}$.
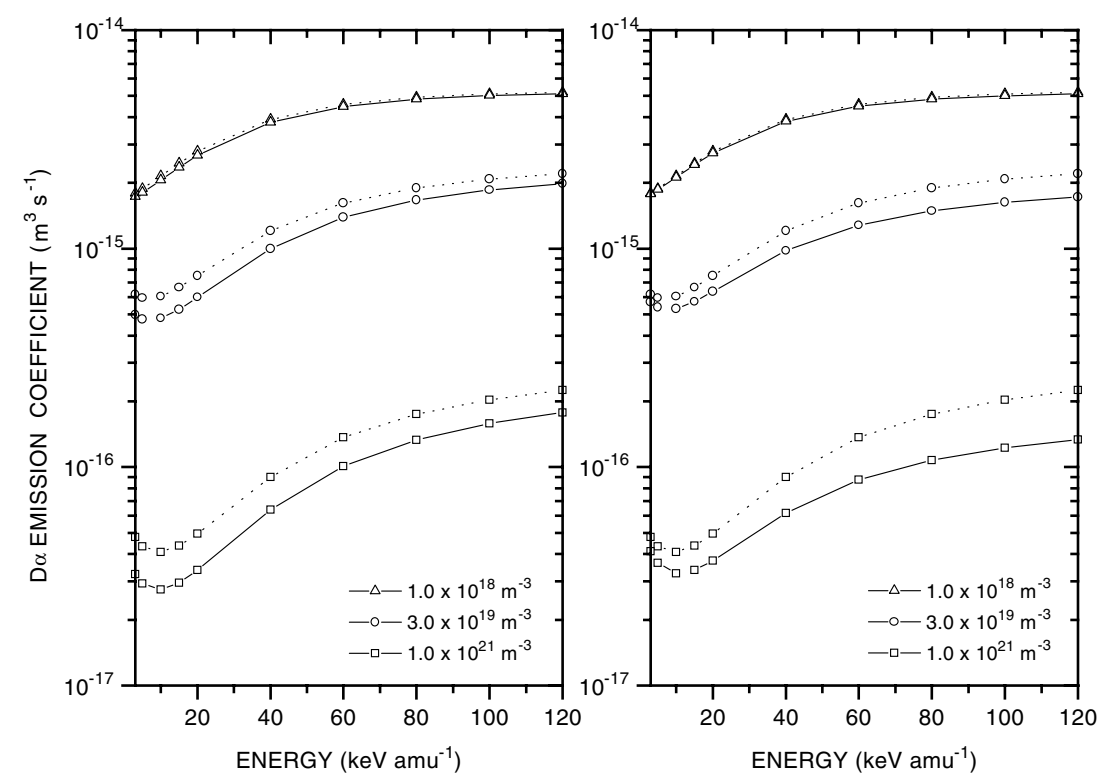

Figure 18. Effective $\mathrm{D}_{\alpha}$ emission coefficient for a pure $\mathrm{D}^{+}$plasma. The figure on the left exhibits the results of increasing the charge exchange and ion impact ionization cross sections from the $n=2,3$ and 4 quantum shells by $50 \%$. The plot on the right shows the influence of increasing the cross sections by $100 \%$ for the transitions $n \rightarrow n^{\prime}$, where $n \in[2,3]$ and $n^{\prime} \in[3,5]$. In each case the broken curves are the results from the unmodified data. $T_{\mathrm{i}}=2.0 \times 10^{3} \mathrm{eV}$. 

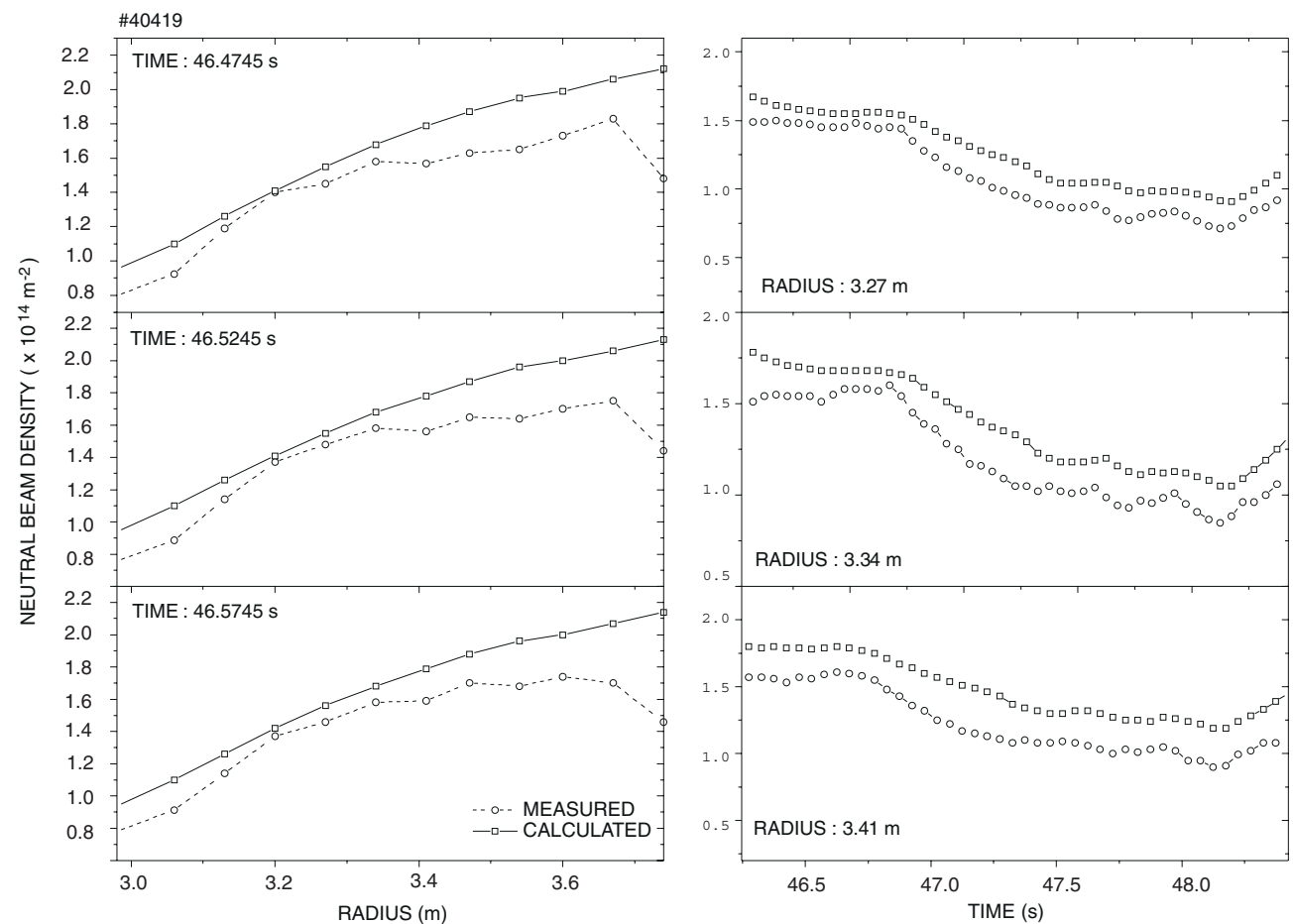

Figure 19. Results obtained for the single beam bank pulses No 40419. The plots on the left shows the radial dependence of the measured and calculated line integrated neutral beam densities at $46.4745,46.5245$ and $46.5745 \mathrm{~s}$. The plots on the right show the time dependence of the measured and calculated line integrated neutral beam densities at the radial positions of $3.27,3.34$ and $3.41 \mathrm{~m}$.

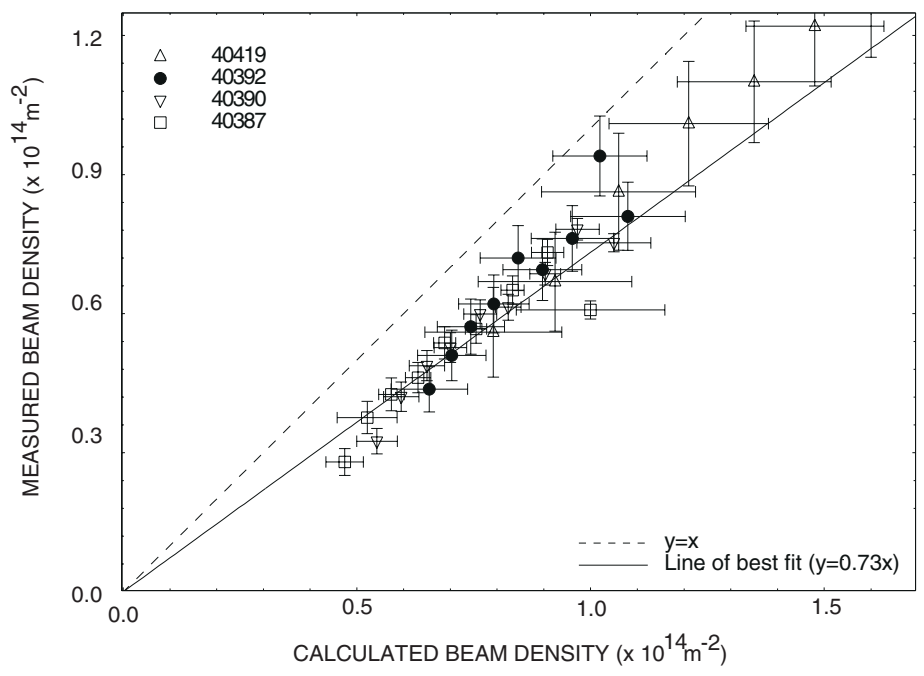

Figure 20. A comparison between the measured and calculated line integrated neutral beam densities for a range of single beam bank pulses. A measure of the deviation between the measured and calculated beam densities is obtained by comparing the full line with that of the broken line, which represents perfect agreement. 


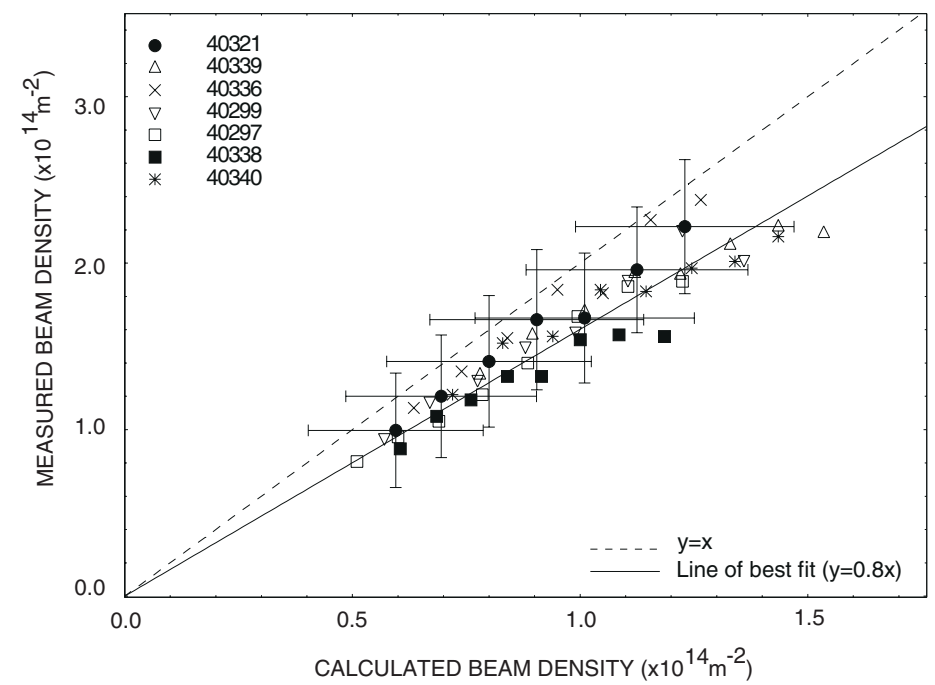

Figure 21. The variation between the measured and calculated line integrated neutral beam densities for a range of double beam bank pulses. The full line represents a line of best fit through the data points. A measure of the deviation between the measured and calculated beam densities is obtained by comparing the full line with that of the broken line, representing perfect agreement.

assigned error bar is simply the standard deviation associated with the time averaged value. Similar results were obtained from the analysis of double beam bank spectra. In figure 21 we show the the variation between the measured and calculated neutral beam densities for a range of double beam bank pulses.

\section{Conclusions}

A critical examination and reworking of the modelling and analysis methods required for systematic deduction of neutral deuterium beam density in fusion plasmas has been presented. The methods are fully integrated with deduction of impurity concentrations by charge exchange spectroscopy and can use either measured beam emission $\mathrm{D}_{\alpha}$ or modelled beam attenuation. The two approaches are consistent within an overall error of $25 \%$. Thus beam emission spectroscopy, charge exchange spectroscopy and beam attenuation can be supported to acceptable precision in all existing and anticipated tokamak conditions.

The study indicates that precision is limited at the fundamental collision data level by the accuracy of impurity ion cross sections for ionization and redistribution of excited deuterium states. The experimental limitation at the JET Joint Undertaking is the precision of reduction and isolation of the integral primary beam fraction emissivity from the observed superposition of $\mathrm{D}_{\alpha}$ Stark multiplet features from double beam banks and fractional energy components. An additional discrepancy at JET, indicating about a $25 \%$ roll-off of the extracted beam emission signal on the outermost observational lines of sights, which exceeds the effect of the measurement localization considerations of section 4.2 , is present and unexplained. Note that the results of the present analysis given in figures 20 and 21 of section 8 incorporate the localisation uncertainty. Estimates also indicate that partially ionized impurities at the lower temperature plasma edge are not the explanation. 
The effective beam stopping and beam emission coefficients have been structured and archived for general diagnostic use within the framework of ADAS. The data structures and derived data retrieval are suited to rapid interpulse analysis. ADAS codes provide an easy interactive re-computation capability for tuning the derived databases to arbitrary beam reference conditions and central plasma parameters. The derived data are comprehensive for mixed impurity plasmas that include any light elements up to neon.

Recent extensions in the fundamental and derived charge exchange effective emission coefficients for excited deuterium donors states (Hoekstra et al 1998), coupled with the present capability for modelling or measuring the $\mathrm{D}(n=2,3, \ldots)$ local populations allow a level of precision in charge exchange spectroscopy at lower beam energies well beyond that previously available.

\section{Acknowledgments}

We would like to thank Dr Shingo Suzuki for providing beam stopping cross section data in tabular form.

\section{References}

Anderson H 1999 PhD Thesis University of Strathclyde, Glasgow

Bell K L, Gilbody H B, Hughes J G, Kingston A E and Smith F J 1982 Culham Laboratory Report CLM-R216

Boileau A, von Hellermann M, Mandl W, Summers H P, Weisen H and Zinoviev A 1989a J. Phys. B: At. Mol. Phys. 22 L145

Boileau A, von Hellermann M, Horton L D, Spence J and Summers H P 1989b Plasma Phys. Control. Fusion 31779 Boley C D, Janev R K and Post D E 1984 Phys. Rev. Lett. 52534

Bray I and Stelbovics A T 1992 Phys. Rev. A 466995

Bray I 1999 private communication

Burgess A and Summers H P 1974 Mon. Not. R. Astron. Soc. 174345

Busnengo H F, Corchs S E, Martinez A E and Rivarola R D 1996 Phys. Scr. T 6288

Callaway J 1994 Atomic Data Nucl. Data Tables 579

Clark R E H, Sampson D H and Goett S J 1982 Astrophys. J. Suppl. Series 49545

Ermolaev A M 1990 J. Phys. B: At. Mol. Phys. 23 L45

Fritsch W and Lin C D 1991 Physics Reports 202 No. 1 and 2

Golden L B, Clark R E H, Goett S J and Sampson D H 1981 Astrophys. J. Suppl. Series. 45603

Hoekstra R, Anderson H, Bliek F W, von Hellermann M G, Maggi C F, Olson R E and Summers H P 1998 Plasma Phys. Control. Fusion $\mathbf{4 0} 1541$

Isler R C 1994 Plasma Phys. Control. Fusion 36171

Janev R K, Boley C D and Post D E 1989 Nucl. Fusion 292125

Janev R K and Krystic P S 1992 Phys. Rev. A 465554

Janev R K and Smith J 1993 Atomic Plasma Mater. Int. Data Fusion 41

Korotkov A A and Samsonov M 1989 Ioffe Physico-technical Institute Report I351

Lodge J G, Percival I C and Richards D 1976 J. Phys. B: At. Mol. Phys. 9239

Mandl W, Wolf R, von Hellermann M and Summers H P 1993 Plasma Phys. Control. Fusion 351371

Morsi H W, von Hellermann M, König R W T and Schröpf H 1995 Plasma Phys. Control. Fusion 371407

Percival I and Richards D 1975 Adv. At. Mol. Phys. 111

Riviere A C 1971 Nucl. Fusion 11363

Sampson D H, Goett S J and Clark R E H 1983 Atomic Data Nucl. Data Tables 29467

Shah M B, Elliot D S and Gilbody H B 1987 J. Phys. B: At. Mol. Phys. 203501

Shah M B and Gilbody H B 1981 J. Phys. B: At. Mol. Phys. 42361

Spence J and Summers H P 1986 J. Phys. B: At. Mol. Phys. 193749

Summers H P 1994 JET Joint Undertaking Report JET-IR(94)06

Summers H P 1999 ADAS User Manual Version 2.1 webpage http:/patiala.phys.strath.ac.uk/adas/ (Glasgow: University of Strathclyde) 
Suzuki S, Shirai T, Nemoto N, Tobita K, Kubo H, Sugie T, Sakasa A and Kumsama Y 1998 Plasma Phys. Control. Fusion 402097

Toshima N and Tawara H 1995 National Institute for Fusion Science Report NIFS-DATA-26 Nagoya, Japan

Vainstein L A, Sobelman I I and Presnyakov L P 1962 Sov. Phys.-JETP 16370

von Hellermann M G and Summers H P 1993 Atomic and Plasma Material Interaction Processes in Contolled

Thermonuclear Fusion ed R K Janev and H W Drawin (Amsterdam: Elsevier)

von Hellermann M G et al 1995 JET Joint Undertaking Report JET-P(95)63

Wagner F et al 1982 Phys. Rev. Lett. 491408

Zwingmann W, O'Brein D P and Barlett D 1997 JET Joint Undertaking Report JET-P(97)57 